\title{
Increased Platelet Deposition on Atherosclerotic Coronary Arteries
}

\author{
G. Henrita van Zanten, ${ }^{\star}$ Sytske de Graaf, ${ }^{\star}$ Pieter J. Slootweg, ${ }^{\star}$ Harry F. G. Heijnen, ${ }^{\star}$ \\ Thomas M. Connolly, ${ }^{3}$ Philip G. de Groot, * and Jan J. Sixma * \\ Departments of ${ }^{*}$ Hematology and ${ }^{\ddagger}$ Pathology, University Hospital Utrecht, 3508 GA Utrecht, The Netherlands; \\ and ${ }^{\S}$ Merck Research Laboratories, West Point, Pennsylvania 19486
}

\section{Abstract}

A ruptured atherosclerotic plaque leads to exposure of deeper layers of the plaque to flowing blood and subsequently to thrombus formation. In contrast to the wealth of data on the occurrence of thrombi, little is known about the reasons why an atherosclerotic plaque is thrombogenic. One of the reasons is the relative inaccessibility of the atherosclerotic plaque. We have circumvented this problem by using $6-\mu \mathrm{m}$ cryostat cross sections of human coronary arteries. These sections were mounted on coverslips that were exposed to flowing blood in a rectangular perfusion chamber. In normal-appearing arteries, platelet deposition was seen on the luminal side of the intima and on the adventitia. In atherosclerotic arteries, strongly increased platelet deposition was seen on the connective tissue of specific parts of the atherosclerotic plaque. The central lipid core of an advanced plaque was not reactive towards platelets. The results indicate that the atherosclerotic plaque by itself is more thrombogenic than the normal vessel wall. To study the cause of the increased thrombus formation on the atherosclerotic plaque, perfusion studies were combined with immunohistochemical studies. Immunohistochemical studies of adhesive proteins showed enrichment of collagen types I, III, V, and VI, vitronectin, fibronectin, fibrinogen/fibrin, and thrombospondin in the atherosclerotic plaque. Laminin and collagen type IV were not enriched. von Willebrand Factor (vWF) was not present in the plaque. The pattern of increased platelet deposition in serial cross sections corresponded best with areas in which collagen types I and III were enriched, but there were also areas in the plaque where both collagens were enriched but no increased reactivity was seen. Inhibition of platelet adhesion with a large range of antibodies or specific inhibitors showed that vWF from plasma and collagen types I and / or III in the plaque were involved. Fibronectin from plasma and fibronectin, fibrinogen, laminin, and thrombospondin in the vessel wall had no effect on platelet adhesion. We conclude that the increased thrombogenicity of atherosclerotic lesions is due to changes in quantity and nature of collagen types I and/or III. (J. Clin. Invest. 1994. 93:615-632.) Key words: atherosclerosis • thrombosis • platelet receptors • vessel wall proteins • perfusion system

This work was presented in abstract form (1991. Thromb. Haemostasis. 65:1114. [Abstr.]).

Address correspondence to Dr. G. H. van Zanten, Department of Hematology, University Hospital Utrecht, P.O. Box 85500, 3508 GA Utrecht, The Netherlands.

Received for publication 4 January 1993 and in revised form 9 October 1993

J. Clin. Invest.

(c) The American Society for Clinical Investigation, Inc.

$0021-9738 / 94 / 02 / 0615 / 18 \quad \$ 2.00$

Volume 93, February 1994, 615-632

\section{Introduction}

Myocardial infarction is in $>90 \%$ of cases caused by an occlusive thrombus in a coronary artery branch (1). The underlying substrate for thrombus formation is usually a ruptured atherosclerotic plaque (2-5). This leads to exposure of deeper layers of the plaque to flowing blood. The atherosclerotic plaque has been assumed to be very thrombogenic, and contact of flowing blood with deeper layers should thus automatically lead to thrombosis. Thrombi on atherosclerotic plaques may cause myocardial infarction and angina, but may also lead to a further increase in atherosclerosis.

Despite the evident importance of this issue, few studies exist about the thrombogenicity of the atherosclerotic plaque. Coagulation tests with tissue extracts of atheromas $(6,7)$ and addition of atheroma suspension to blood in a rotating tilted closed plastic tube ("Chandler loop" model) (7-9) tended to show less thrombogenicity than expected $(6,8,9)$. More recently histochemical studies showed that there is more tissue factor in an atherosclerotic plaque than in the normal vessel wall $(10,11)$. Perfusion studies with blood over hyperlipidemic rabbit atherosclerotic aortic subendothelium showed decreased platelet adhesion (12), whereas the injured artificially created neointima in rabbits was more thrombogenic than the injured normal intima (13). A problem of most studies is that the relevant deeper layers of the atherosclerotic plaque are relatively inaccessible to flowing blood. Artificial rupture of the atherosclerotic plaque in order to bring blood in contact with deeper layers has the disadvantage that it is difficult to evaluate the actual thrombogenicity because of the disturbed blood flow pattern. We have circumvented these problems by using cryostat cross sections of postmortem human coronary arteries. These cross sections were mounted on coverslips and anticoagulated blood was perfused over them in a rectangular perfusion chamber (14). These studies showed platelet deposition and thrombus formation particularly on the luminal side of the subendothelium and on the adventitia in normal vessels and strongly increased platelet deposition on the connective tissue of the atherosclerotic plaque. No platelet deposition was seen on the central lipid core of the atherosclerotic plaque.

To study the cause of increased platelet deposition on the atherosclerotic plaque, perfusion studies over cross sections of atherosclerotic coronary arteries were combined with immunohistochemical studies and inhibition studies using antibodies and specific inhibitors.

\section{Methods}

\section{Specimens}

Postmortem coronary arteries from 25 patients with different causes of death and fresh coronary arteries from 11 patients who were undergoing cardiac transplantation were obtained from the Departments of Pathology and Cardiology (University Hospital Utrecht) (Table I). 
The postmortem coronary arteries were collected within $24 \mathrm{~h}$ of death. The age of deceased patients ranged from 22 to $79 \mathrm{yr}$ with a mean of 54 $\mathrm{yr}$. The age of heart transplant recipients ranged from 33 to $55 \mathrm{yr}$ with a mean of 45 yr. Patients with severely calcified coronary arteries were excluded. Coronary segments, $\sim 1 \mathrm{~cm}$ in length, of the subepicardial right, left circumflex, and left anterior descending arteries were rinsed in Hepes-buffered saline (HBS; ${ }^{1} 10 \mathrm{mM}$ Hepes, $145 \mathrm{mM} \mathrm{NaCl}$ ), $\mathrm{pH}$ 7.35. Representative segments of coronary arteries were divided into two unequal parts. The smallest part was formalin fixed and embedded in paraffin for morphology. The other part together with undivided segments were embedded in optimal cutting temperature compound (Miles Inc., Elkhart, IN), snap frozen in liquid nitrogen, and stored at $-70^{\circ} \mathrm{C} .108$ different frozen coronary segments were used for perfusion studies: 28 normal segments from 10 patients without atherosclerosis, and 80 segments from 26 patients with atherosclerosis. The latter 80 segments could be divided into 14 normal segments and 66 atherosclerotic segments. Coronary arteries from autopsies in three newborn babies and arteries from three umbilical cords were also used.

\section{Perfusion studies}

Perfusions were carried out in a rectangular perfusion chamber, the characteristics of which have been described previously $(14,15)$. Glass coverslips ( $18 \times 18 \mathrm{~mm}$; Menzel Gläser, Braunschweig, Germany) were cleaned by soaking overnight in chromosulfuric acid and rinsing thoroughly with deionized water. They were air dried, coated with Denhardt's solution $(0.02 \%$ Ficoll 70 [Pharmacia AB, Uppsala, Sweden], $0.02 \%$ BSA fraction $\mathrm{V}$, and $0.02 \%$ polyvinylpyrrolidone 360 [Sigma Chemical Co., St Louis, MO] in deionized water) for $3 \mathrm{~h}$ at $68^{\circ} \mathrm{C}$. The coverslips were rinsed with deionized water $(20 \mathrm{~s})$ and fixed in ethanol/acetic acid $(3: 1 ; 20 \mathrm{~min}$ at room temperature), then air dried and baked for $3 \mathrm{~h}$ at $180^{\circ} \mathrm{C}$.

Frozen cross sections of arteries at $6-\mu \mathrm{m}$ thickness were cut at $-20^{\circ} \mathrm{C}$ and were mounted on the Denhardt-coated glass coverslips. The Denhardt coating was used to prevent detachment of the tissue section in flow. Tissue sections stick very well on this coating. After air drying $(2 \mathrm{~h})$, the sections were incubated in a $4 \%$ human albumin (Behringwerke AG, Marburg, Germany) solution in HBS (12-16 h at $\left.4^{\circ} \mathrm{C}\right)$ to block aspecific binding. Almost no platelets adhered to the albumin-blocked Denhardt surface. The few platelets that adhered were platelets in the contact phase. The coverslips were rinsed with HBS and were inserted into the perfusion chamber. A minimum of six serial sections per frozen artery segment were used for perfusions. Fresh blood from normal donors who denied having taken aspirin in the preceding $10 \mathrm{ds}$ was anticoagulated with $1: 10 \mathrm{vol}$ of $110 \mathrm{mM}$ trisodium citrate, or with 1:10 vol of Orgaran (a low molecular weight heparinoid; kindly provided by Organon, Oss, The Netherlands) in saline (final concentration, $15 \mathrm{U} / \mathrm{ml}$ ). The choice of anticoagulant was dependent on the experiment; Orgaran was used, since we had recently observed that adhesion to collagen type IV was almost absent and adhesion to collagen type I was decreased in citrated blood at high shear rate. Platelet adhesion under nonpulsatile flow conditions was performed using a rectangular perfusion chamber as described $(14,15)$. Briefly, duplicate glass coverslips with cryostat sections were inserted in the chamber. Whole blood, $15 \mathrm{ml}$, was prewarmed at $37^{\circ} \mathrm{C}$ for $5 \mathrm{~min}$ and then recirculated through the chamber for $5 \mathrm{~min}$ at a wall shear rate of $1,600 / \mathrm{s}$, corresponding to the somewhat increased shear rate in narrowed coronary arteries (16). The system was rinsed with HBS after each perfusion. The coverslips were removed from the chamber, rinsed with HBS, and then fixed in acetone (10 $\mathrm{min}$ ) or paraformaldehyde (2\% in PBS, $\mathrm{pH} 7.4, \sim 5 \mathrm{~min}$ )

Microaggregate formation during recirculation of the blood was measured as described (17). Briefly, duplicate blood samples were taken after recirculation. One of the samples $(111 \mu \mathrm{l})$ was added to $1 \mathrm{ml}$

1. Abbreviations used in this paper: Abs, antibodies; ECM, endothelial cell matrix; Gp, glycoprotein; HBS, Hepes-buffered saline; IEL, internal elastic lamina; LMWH, low molecular weight heparin; r-LAPP, recombinant leech anti-platelet protein; SEM, scanning electron microscopy; SMC, smooth muscle cell; vWF, von Willebrand Factor. of $0.5 \%$ glutardialdehyde (Fluka AG, Buchs, Switzerland) in PBS and the other sample to $1 \mathrm{ml}$ of $4.8 \mathrm{mM}$ dipotassium EDTA (Fluka AG) in PBS. Platelet counts were measured with a platelet analyzer (810; Baker Instruments, Allentown, PA) with apertures set between 3.1 and $16 \mu \mathrm{m}$. Platelet counts before perfusion were between 150,000 and $300,000 / \mu$ l. Microaggregate formation was determined by the following formula: $100 \times$ (platelet count in EDTA sample - platelet count in glutardialdehyde sample) platelet count in EDTA sample. Microaggregate formation was usually $<30 \%$, independent of the tissue sections on the coverslip.

Blood was anticoagulated with $20 \mathrm{U} / \mathrm{ml} \mathrm{low}$ molecular weight heparin (LMWH Fragmin ${ }^{\star}$; Kabi Vitrum, Stockholm, Sweden) plus 20 $\mathrm{U} / \mathrm{ml}$ desulphato-hirudin (a gift from Dr. R. Wallis, Ciba Geigy, Horsham, UK) in studies to find out whether the results could have been influenced by the use of citrate as anticoagulant. Thrombin formation during perfusion was prevented by this anticoagulant mixture as was evident from the fibrinopeptide A values of $<2 \mathrm{ng} / \mathrm{ml}$. Fibrinopeptide A was assayed with a radioimmunoassay kit (Byk-Sangtec Diagnostica, Dietzenbach, Germany) according to the instructions of the manufacturer.

Visualization of platelet deposition. Paraformaldehyde-fixed cross sections were immersed in $0.1 \mathrm{M}$ glycine in $0.05 \mathrm{M}$ Tris-buffered saline (TBS) for $10 \mathrm{~min}$, and rinsed in deionized water for $30 \mathrm{~s}$. Coverslips with acetone- or paraformaldehyde-fixed cross sections were soaked for a few minutes in TBS ( $\mathrm{pH} 7.6$ ) plus $0.05 \%$ Tween-20 (TBS-Tween). Adhered platelets and platelet thrombi were visualized by staining with a biotinylated mAb against glycoprotein (Gp) Ib (mAb $6.74 \alpha$-Ib obtained from Dr. M. Metzelaar, Department of Hematology). This $\mathrm{mAb}$ reacted with the $\mathrm{Gp} \mathrm{Ib} \alpha$-band in a Western blot and gave completely negative results in cytofluorography with platelets of a patient with the Bernard Soulier Syndrome. The biotinylated mAb $6.74 \alpha$-Ib $(0.5 \mu \mathrm{g} / \mathrm{ml}$ in TBS $+1 \%$ normal rabbit serum $)$ was incubated on sections in a humidified box for $60 \mathrm{~min}$ at room temperature. This step was followed by three washes of $5 \mathrm{~min}$ with TBS-Tween. The sections were subsequently incubated with avidin-biotin-horseradish peroxidase complex (Vector Laboratories, Burlingame, CA) for $30 \mathrm{~min}$ at room temperature at a 1:1 ratio, as supplied by the manufacturer, followed by three TBS washes of $5 \mathrm{~min}$. Bound peroxidase was detected by incubation with the chromogenic substrate 3,3'-diaminobenzidine (DAB; Sigma Chemical Co). $50 \mathrm{mg}$ DAB was diluted in $100 \mathrm{ml} 0.1 \mathrm{M}$ phosphate buffer, $\mathrm{pH} 7.35$, to which had been added $33 \mu \mathrm{l} 30 \% \mathrm{H}_{2} \mathrm{O}_{2}$, $0.025 \%$ cobalt chloride, and $0.020 \%$ nickel ammonium sulfate. Cobalt chloride and nickel ammonium sulfate were added to yield a more intense, black reaction product (18). After $10 \mathrm{~min}$, sections were rinsed in deionized water, dehydrated, and mounted. In control sections the first antibody was either omitted or substituted by an irrelevant antibody of similar isotype ( $\mathrm{mAb}$ against pollen of birch; Central Laboratory Blood Transfusion, Amsterdam, The Netherlands).

To determine the cellular content of the perfused cross sections, serial sections were treated as described above with the following $\mathrm{mAbs}$ : mAb anti-Desmin (clone DE-R-11; Dakopatts, Glostrup, Denmark), $\mathrm{mAb}$ anti- $\alpha$-actin (clone asm 1; Boehringer Mannheim GmbH, Mannheim, Germany), mAb anti-human macrophage (clone EBM11; Dakopatts), mAb Leu-M3 (specific for monocytes and macrophages; CD14; Becton Dickinson \& Co., Mountain View, CA), mAb Leu-4 (specific for pan T cells; CD3; Becton Dickinson \& Co.), and mAb anti-human leukocyte common antigen (CD45; Dakopatts). Since these mAbs were not labeled with biotin, an incubation step with a biotinylated rabbit anti-mouse antibody (Dakopatts) was inserted ( $1: 400$ diluted in TBS $+1 \%$ normal rabbit serum, $30 \mathrm{~min}$ at room temperature).

Evaluation of platelet deposition. Platelet deposition on perfused cross sections of (coronary) arteries was evaluated by light microscopy. Beside this qualitative evaluation, 23 sections of 6 different coronary segments were analyzed morphometrically with the Interaktiv Bild Analyse System (IBAS) image analysis system (Zeiss/Kontron, Eching, Germany). For this evaluation, microscopical sections stained for platelets were scanned with a charge coupled device camera. The images were digitized 10 times and averaged to improve signal/noise 
ratio (frame size: $512 \times 512$ pixels, 8 bits $/$ pixel $=256$ grey levels). Before analysis, the images were corrected for shading and contrast transitions were enhanced. After marking the intima and the media, the platelet coverage of these layers was determined by adjustment of the right grey level at a magnification of 4 ( Table II). Some locations of the intima and the media were enlarged 16 times, after which areas of platelets or thrombi were determined, as well as the total platelet coverage (Tables III and IV). The total platelet coverage, evaluated with the IBAS image analysis system, was the same as that evaluated with the image analyzer (40-10; AMS Ltd., Saffron Walden, UK) normally used in our laboratory (results not shown). The data presented in Tables II-IV were expressed as mean \pm SEM (Tables II and III) or as mean \pm SD (Table IV). The data were compared using the $t$ test. Areas of platelets or thrombi were defined as the areas that were covered by platelets or thrombi. The total platelet coverage of a location was defined as the percentage of the surface covered by platelets and platelet thrombi.

Scanning electron microscopy (SEM). For SEM, 6- $\mu \mathrm{m}$ cryostat sections were transferred to Thermanox ${ }^{\circledR}$ coverslips (Miles, Inc., Naperville, IL). After perfusion, sections were fixed in $2 \%$ glutaraldehyde in PBS, pH 7.4. The sections were postfixed in $1 \%$ osmium tetroxide for 1 $\mathrm{h}$, dehydrated in a graded series of ethanol, and dried by the critical point procedure, using $\mathrm{CO}_{2}$ as transitional fluid. The samples were sputter coated with a thin layer of gold and viewed in a SEM (Cam Scan S2, Cambridge, UK).

\section{Immunohistochemical studies}

To identify the vessel wall components involved in platelet-vessel wall interactions, specific antibodies (Abs) for immunolocalization of adhesive proteins were used: mAb RU2 against the A2-repeat of von Willebrand Factor (VWF; University Hospital Utrecht), mAb antilaminin (clone 2G6/A2; Boehringer Mannheim GmbH), mAb antithrombospondin (Sigma Chemical Co.), $\mathrm{F}\left(\mathrm{ab}^{\prime}\right)_{2}$ fragment of the polyclonal $\mathrm{Ab}$ against fibronectin (Cappel Laboratories, Cochranville, $\mathrm{NC}$ ), monospecific polyclonal Ab against vitronectin (kindly provided by Dr. K. T. Preissner, Max-Planck-Gesellschaft, Bad Nauheim, Germany), polyclonal $\mathrm{Ab}$ anti-fibrinogen (Cappel Laboratories), affinitypurified polyclonal Abs against collagen types I and V (Southern Biotechnology Ass., Birmingham, AL), and mAbs against collagen types III, IV, and VI (Heyl, Berlin, Germany).

Serial 6- $\mu \mathrm{m}$ cross sections of coronary arteries were cut in a cryostat $\left(-20^{\circ} \mathrm{C}\right)$ and were mounted on clean glass slides. After air drying, the slides were fixed in acetone or paraformaldehyde and were handled as described earlier. Dependent on the first Ab, biotinylated rabbit antimouse (Dakopatts), biotinylated goat anti-rabbit, and horse anti-goat (Vector Laboratories, Burlingame, CA) was used as second Ab. 13 coronary segments with atherosclerotic plaque from 8 patients, and 7 unaffected coronary segments from 4 patients were examined.

\section{Inhibition studies}

In a further attempt to identify the components responsible for platelet deposition on the atherosclerotic vessel wall, we tried to inhibit platelet-vessel wall interactions. A total of 21 coronary segments with atherosclerotic plaque from 12 patients were examined. The results were obtained in three independent experiments.

Antibodies. mAb CLB-RAG35 against the platelet binding domain on vWF was kindly provided by Dr. J. A. van Mourik (Central Laboratory of the Netherlands Red Cross Blood Transfusion Service, Amsterdam, The Netherlands). This Ab has been described previously (19). The amount added to the perfusate was determined before perfusion in a ristocetin-induced platelet aggregation (RIPA) assay. A 10 times excess was used for perfusion experiments. CLB-RAG35 was used both as ascites and IgG.

$\mathrm{F}\left(\mathrm{ab}^{\prime}\right)_{2}$ fragment of the polyclonal Ab against fibronectin was used (Cappel Laboratories). This Ab completely inhibited platelet adhesion to purified fibronectin (Sara Beumer, University Hospital Utrecht, personal communication ) and was used in previous studies (20,21). Tissue sections on glass coverslips were preincubated with $100 \mu 11.5 \mathrm{mg}$ / $\mathrm{ml} \mathrm{Ab} \mathrm{(60} \mathrm{min} \mathrm{at} \mathrm{room} \mathrm{temperature).}$
mAb AK 2 (ascites) against the vWF-binding site of Gp Ib was kindly provided by Dr. M. C. Berndt (22) (Baker Institute, Melbourne, Australia). The amount added to the perfusate was determined in a RIPA assay. A 10 times excess was used for perfusion experiments. As a control mAb AK 3 (ascites), also against Gp Ib but not against the vWF-binding site, was used (also kindly provided by Dr. M. C. Berndt).

mAb GoH3 (ascites) against VLA-6 (23) was kindly provided by Dr. A. Sonnenberg (Central Laboratory of the Netherlands Red Cross Blood Transfusion Service). $2 \mu \mathrm{l}$ ascites $/ \mathrm{ml}$ plasma was added to the perfusate. This concentration completely inhibited platelet adhesion on purified laminin (24). Antibodies were added to the perfusates 30 min before perfusion.

Peptides/proteins. D-arginyl-glycyl-L-aspartyl-L-tryptophan peptide against the RGD binding site of Gp IIb-IIla (Rhône-PoulencRorer, Paris, France) was added to the perfusate ( 12.5 and $100 \mu \mathrm{M})$.

A recombinant leech anti-platelet protein $(r-\operatorname{LAPP})(25,26)$ that binds specifically to collagen inhibits collagen-induced platelet aggregation and platelet adhesion to collagen surfaces. The effect of r-LAPP

Table I. Patient Data

\begin{tabular}{|c|c|c|c|}
\hline Number & Age & Sex & Disease \\
\hline & $y r$ & & \\
\hline $\mathrm{H} 01^{*}$ & 53 & $\mathrm{~m}$ & Brain tumor \\
\hline $\mathrm{H} 03$ & 63 & f & Myocardial infarction \\
\hline $\mathrm{H} 04$ & 45 & $\mathrm{f}$ & Intoxication \\
\hline H06 & 79 & $\mathrm{f}$ & Aortic aneurysm \\
\hline $\mathrm{H} 08$ & 51 & $\mathrm{~m}$ & Leukemia \\
\hline $\mathrm{H} 10^{*}$ & 36 & $\mathrm{f}$ & Cardiomyopathy \\
\hline H11 & 78 & $\mathrm{f}$ & Cerebral lymphoma \\
\hline $\mathrm{H} 12$ & 77 & $\mathrm{~m}$ & Gastric carcinoma \\
\hline $\mathrm{H} 14$ & 67 & $\mathrm{~m}$ & Cerebral hemorrhage \\
\hline $\mathrm{H} 20$ & 53 & f & Aortic aneurysm \\
\hline $\mathrm{H} 23$ & 55 & $\mathrm{~m}$ & Cerebral trauma \\
\hline $\mathrm{H} 24$ & 71 & $\mathrm{~m}$ & Aortic aneurysm \\
\hline $\mathrm{H} 26^{*}$ & 63 & $\mathrm{~m}$ & Aortic aneurysm \\
\hline $\mathrm{H} 27^{*}$ & 48 & $\mathrm{f}$ & Lymphoma \\
\hline $\mathrm{H} 29$ & 39 & $\mathrm{~m}$ & Septicemia \\
\hline $\mathrm{H} 31$ & 43 & f & Septicemia \\
\hline $\mathrm{H} 32$ & 66 & f & Cerebral lymphoma \\
\hline $\mathrm{H} 33$ & 45 & f & Ovarium carcinoma \\
\hline $\mathrm{H} 37^{* \ddagger}$ & 35 & f & Myocardial infarction \\
\hline $\mathrm{H} 38^{*}$ & 22 & $\mathrm{~m}$ & Traffic accident \\
\hline $\mathrm{H} 41$ & 33 & $\mathrm{~m}$ & Cerebral hemorrhage \\
\hline $\mathrm{H} 44^{\ddagger}$ & 55 & $\mathrm{~m}$ & Cardiomyopathy \\
\hline $\mathrm{H} 45$ & 61 & $\mathrm{~m}$ & Lung carcinoma \\
\hline H46 & 75 & $\mathrm{~m}$ & Myocardial ischaemia \\
\hline $\mathrm{H} 48$ & 46 & $\mathrm{~m}$ & Myocardial infarction \\
\hline H49* & 28 & f & Pulmonary embolism \\
\hline $\mathrm{H} 5 \mathrm{I}^{\ddagger}$ & 50 & $\mathrm{~m}$ & Cardiomyopathy \\
\hline $\mathrm{H} 53^{\ddagger}$ & 45 & $\mathrm{~m}$ & Cardiomyopathy \\
\hline $\mathrm{H} 57^{\ddagger}$ & 55 & f & Cardiomyopathy \\
\hline $\mathrm{H} 58^{\ddagger}$ & 55 & $\mathrm{~m}$ & Cardiomyopathy \\
\hline $\mathrm{H} 59^{\ddagger}$ & 33 & $\mathrm{f}$ & Cardiomyopathy \\
\hline $\mathrm{H} 60$ & 63 & $\mathrm{f}$ & Lung carcinoma \\
\hline $\mathrm{H} 61^{* \ddagger}$ & 39 & $\mathrm{~m}$ & Valve failure \\
\hline H62* & 40 & $\mathrm{~m}$ & Cardiomyopathy \\
\hline $\mathrm{H} 63^{* \ddagger}$ & 36 & $\mathrm{~m}$ & Cardiomyopathy \\
\hline H64* & 55 & $\mathrm{f}$ & Cardiomyopathy \\
\hline
\end{tabular}

* Patients without atherosclerosis. ${ }^{\ddagger}$ Explanted hearts of heart transplant recipients. 

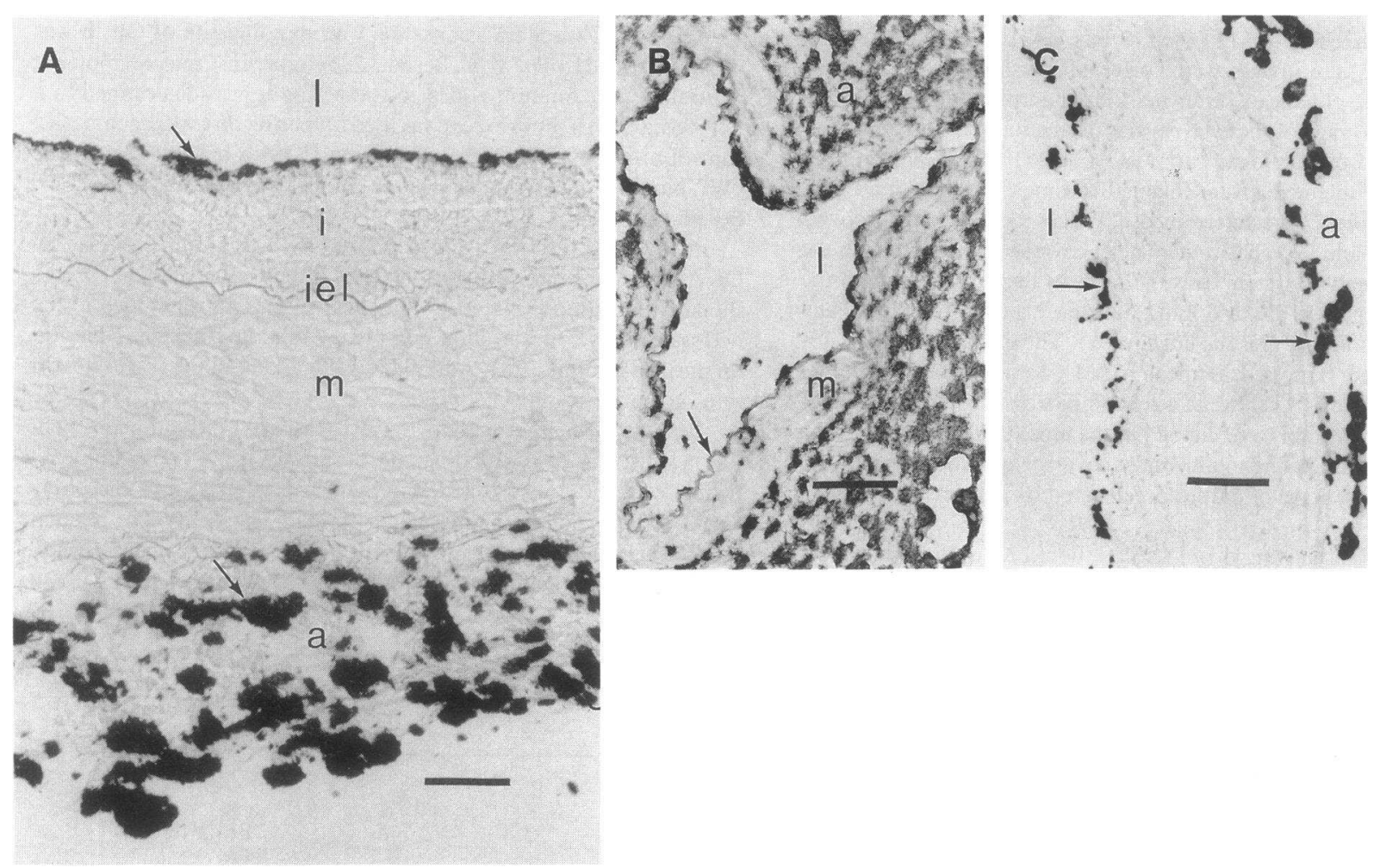

Figure 1. (A) Section of a normal coronary artery after perfusion. Platelet aggregates on the luminal side of the intima $(i)$ and on the adventitia $(a)$ are stained black (arrows). $m$, media; iel, internal elastic lamina; $l$, lumen. Bar $=80 \mu \mathrm{m} .(B)$ Coronary artery from a newborn after perfusion counterstained with Hematoxylin. The layer between endothelial cells and internal elastic lamina is very thin. It appears that platelet aggregates adhere on the IEL (arrow). $l$, lumen; $m$, media; $a$, adventitia plus surrounding tissue. Bar $=130 \mu \mathrm{m}$. ( $C$ ) Umbilical cord artery after perfusion counterstained with Hematoxylin. Platelet aggregates on the luminal side of the vessel wall and on the adventitia $(a)(a r r o w s) . l$, lumen. Bar $=$ $95 \mu \mathrm{m}$.

on platelet adhesion under flow conditions is described in detail elsewhere (Van Zanten, G. H., T. M. Connolly, M. E. Schiphorst, S. De Graaf, P. J. Slootweg, and J. J. Sixma, manuscript submitted for publication). dRGDW and r-LAPP were added to the perfusates $15 \mathrm{~min}$ before perfusion.
Fibronectin-free, $v W F$-free, and patient blood. Platelets and red blood cells were washed by centrifugation of whole blood from a normal donor as previously described (27) and then resuspended in fibronectin-free or vWF-free plasma. Fibronectin-free plasma was obtained by passing normal plasma over a gelatin-Sepharose column as previ-

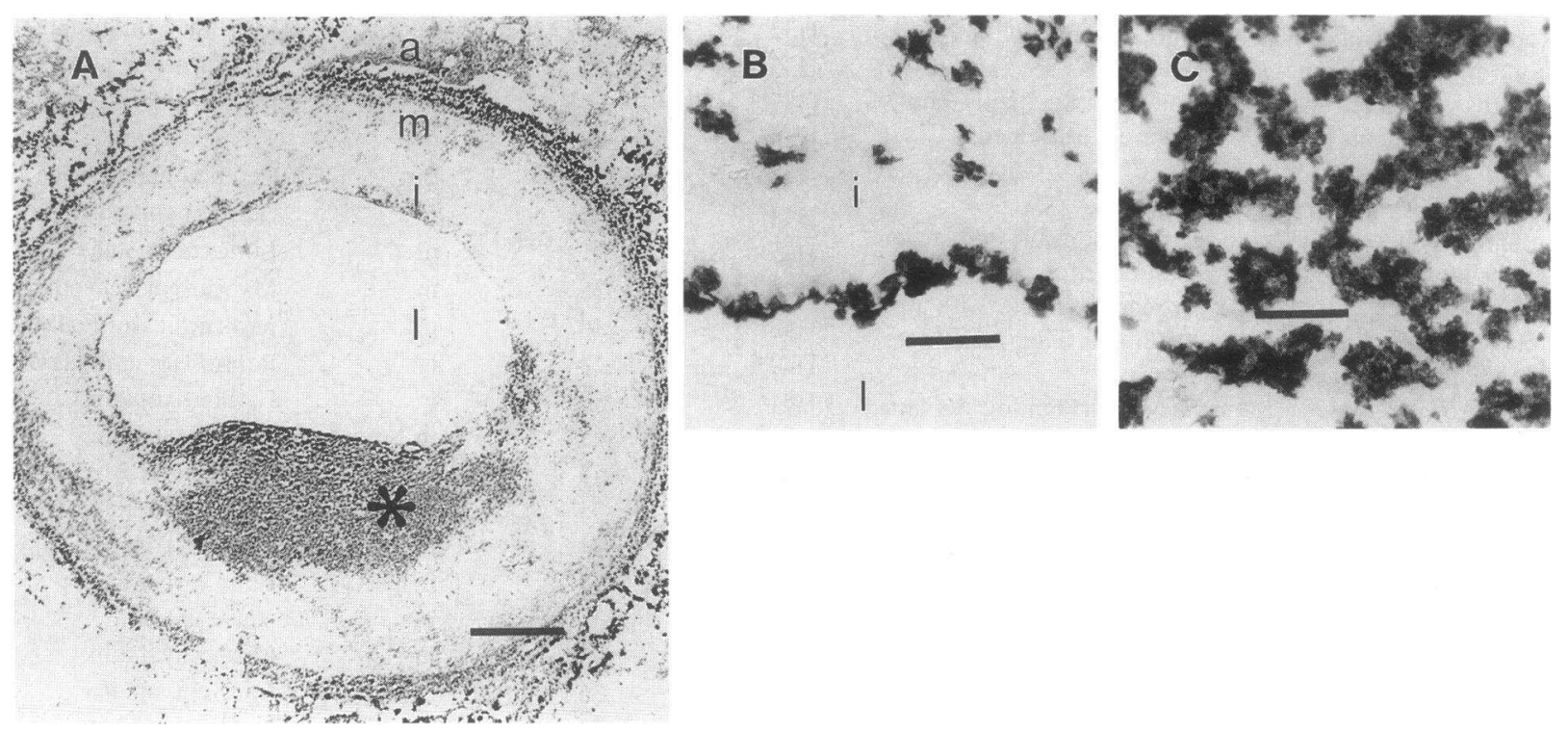

Figure 2. (A) Overview of a coronary artery with an eccentric plaque (*) that is very reactive towards platelets. $l$, lumen; $i$, intima; $m$, media; $a$, adventitia. Bar $=800 \mu \mathrm{m}$. (B) Magnification of $A$. Section of the normal intima $(i)$ opposite the atherosclerotic plaque. $l$, lumen. Bar $=35 \mu \mathrm{m}$. (C) Magnification of $A$. Section of the atherosclerotic plaque. Bar $=35 \mu \mathrm{m}$. 

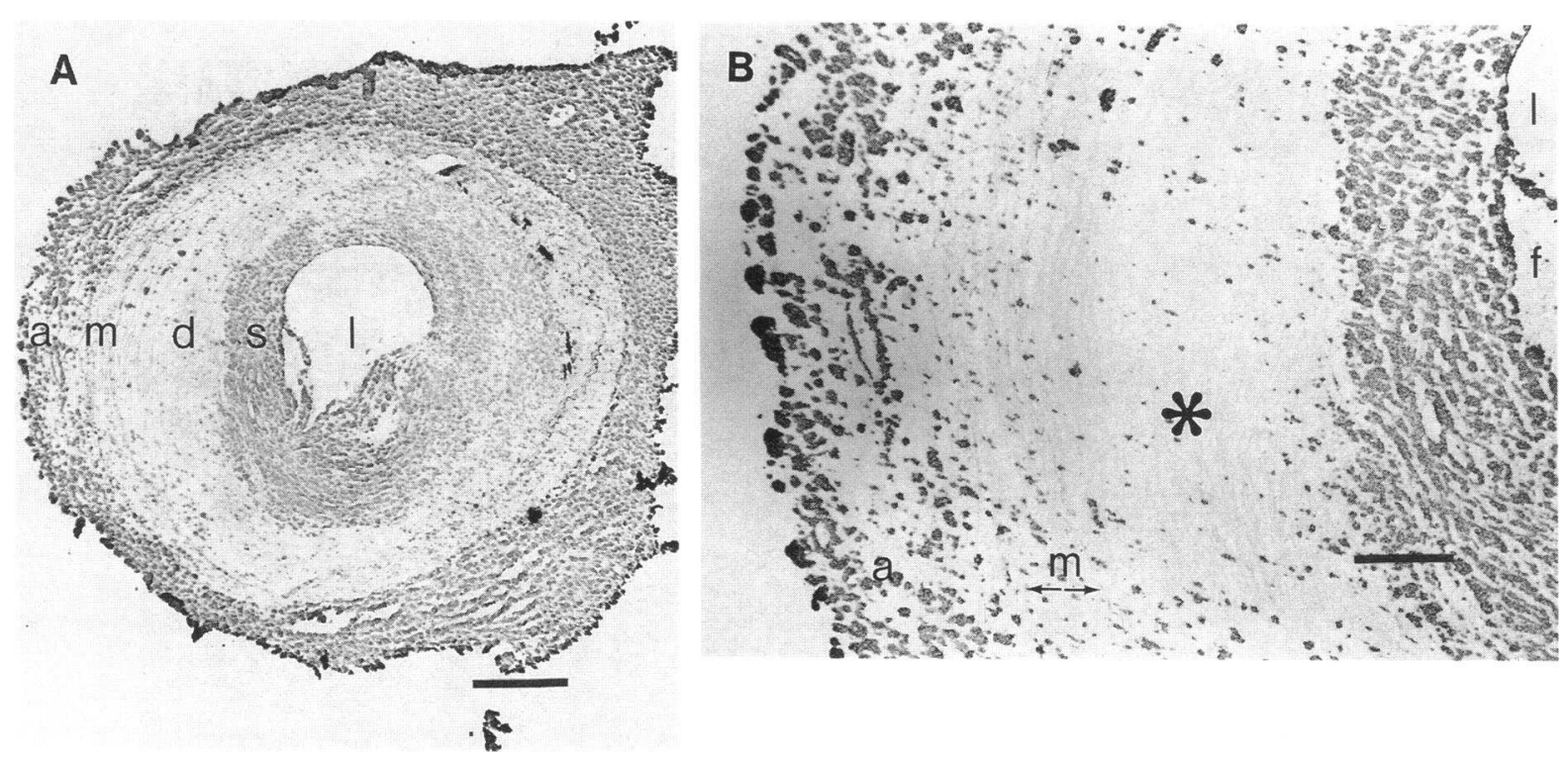

Figure 3. $(A)$ Overview of a coronary artery with a collagen-rich concentric plaque in which the superficial part $(s)$ of the plaque is more reactive towards platelets than the deeper part $(d)$ of the plaque. $l$, lumen; $m$, media; $a$, adventitia. Bar $=800 \mu \mathrm{m}$. $(B)$ Magnification of $A$. Collagen bundles are clearly seen in the plaque $(*) . l$, lumen; $m$, media; $a$, adventitia; $f$, region with foam cells. Bar $=240 \mu \mathrm{m}$.

ously described by Houdijk et al. (20). vWF-free plasma was obtained by affinity chromatography with mAb RU1 (University Hospital Utrecht) coupled to CNBr-Sepharose 4B (Pharmacia, Uppsala, Sweden). The final $\mathrm{vWF}$ content was $<0.05 \mathrm{U} / \mathrm{ml}$ plasma as measured by ELISA. Control perfusates were prepared by resuspending washed platelets and washed red blood cells in their own plasma. Platelet counts were standardized at $200,000 / \mu$ l (final concentration); the hematocrit was between 0.37 and 0.39 . Whole blood was from a patient whose platelets lacked $80 \%$ of $\mathrm{Gp}$ la (28).

EDTA treatment of tissue. Some cross sections of coronary arteries were treated with $10 \mathrm{mM}$ EDTA in HBS $+1 \%$ human albumin (two times for $1 \mathrm{~h}$ at room temperature) before exposure to blood.

Control surfaces. Endothelial cell matrices on glass coverslips were derived from human umbilical vein endothelial cells as previously described (29).

\section{Results}

\section{Perfusion studies}

No differences in reactivity towards blood platelets were observed between postmortem and fresh coronary arteries. The first perfusion experiments were performed with citrated blood. However, to exclude an effect of citrate on platelet adhesion, we performed perfusion experiments with blood anticoagulated with a combination of the LMWH Fragmin and desulphato-hirudin. Six coronary segments from four patients were used for these experiments. Although the platelet aggregates were smaller and less compact, the pattern of platelet deposition was similar to that of the perfusion experiments with blood
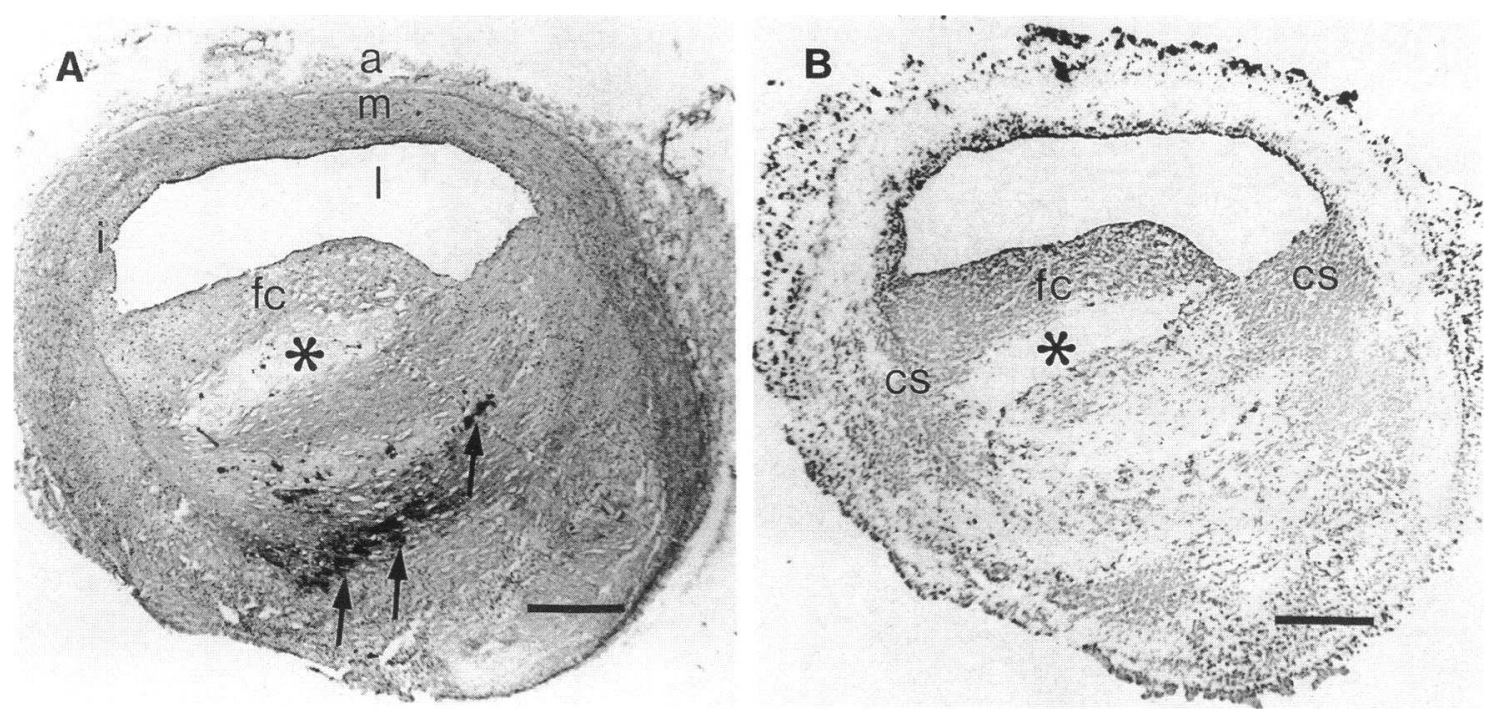

Figure 4. $(A)$ Coronary artery with an eccentric plaque without perfusion, stained with Hematoxylin and Eosin. The fibrous cap $(f \mathcal{C})$ consists of loose connective tissue. A lipid-filled area $(*)$ is seen under the fibrous cap, whereas dense relative acellular connective tissue is seen under the lipid-filled area. Calcium deposits (arrows) are stained black. $l$, lumen; $i$, normal intima; $m$, media; $a$, adventitia. Bar $=500 \mu \mathrm{m}$. $(B)$ Same coronary artery as shown in $A$, but after perfusion. The fibrous cap $(f c)$ and the cellular sides $(c s)$ of the plaque are more reactive towards platelets than the underlying dense connective tissue. The lipid-filled area (*) is not reactive. $B a r=500 \mu \mathrm{m}$. 

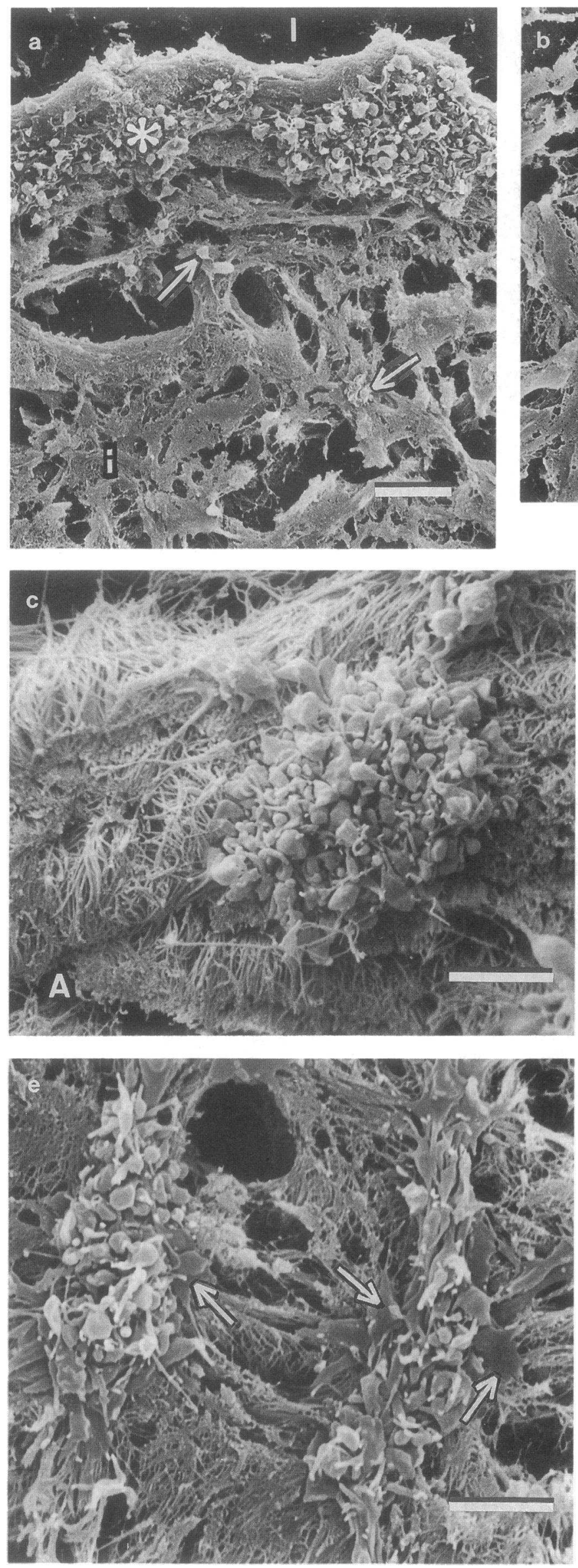
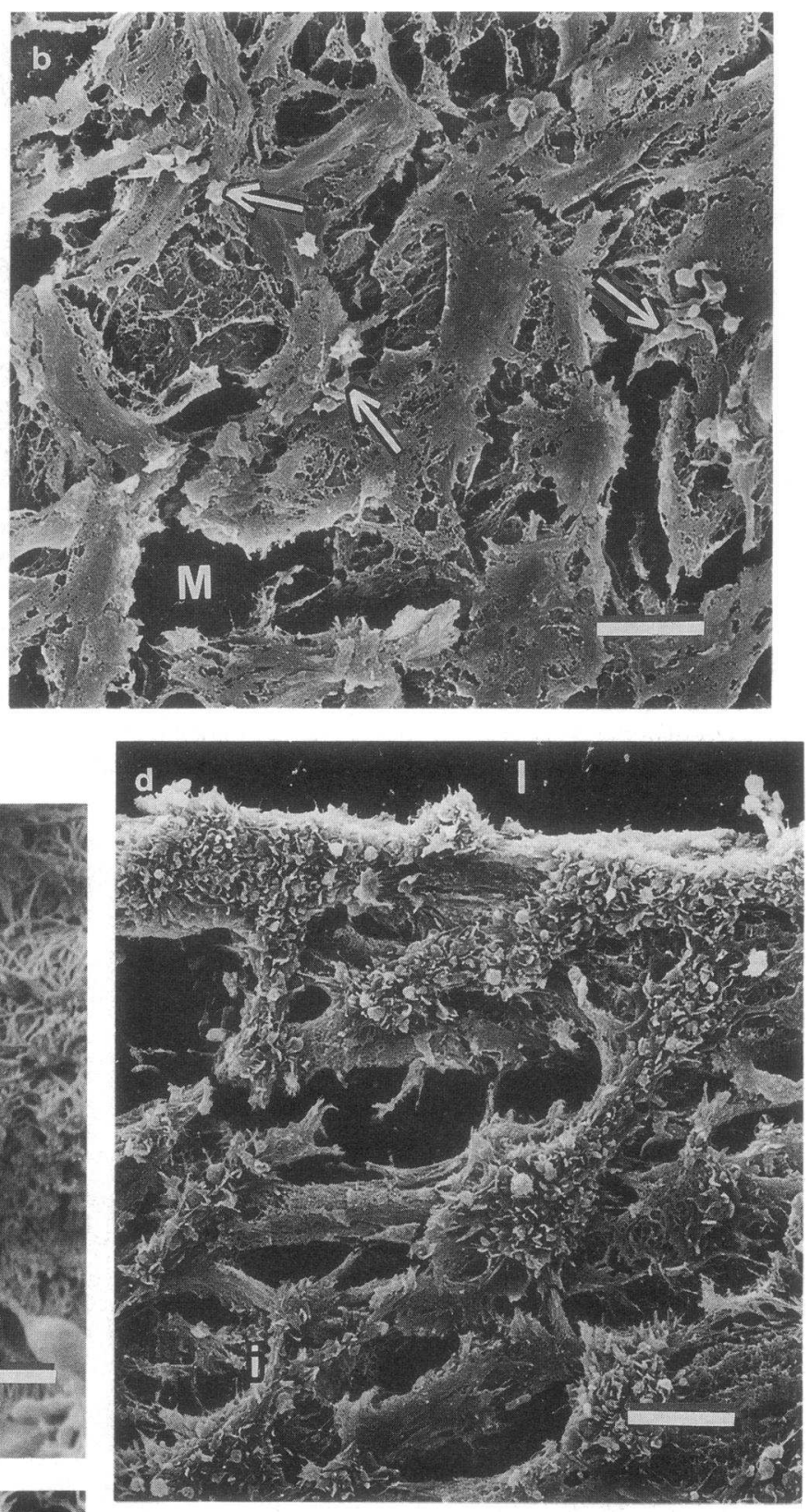
anticoagulated with citrate. In later perfusion experiments we chose the low molecular weight heparinoid Orgaran. Perfusions with Orgaran-anticoagulated blood gave same patterns of platelet deposition as perfusions with citrated blood, whereas the size of aggregates was not diminished as with Fragmin-anticoagulated blood.

Normal coronary arteries. In adults, the layer between the lumen of the vessel and the internal elastic lamina (IEL) was diffusely thickened. The thickness of the intima was often similar to that of the media or exceeded it, in contrast to the intima in newborns in whom the endothelial cells rested almost directly on the IEL. The thickened intima in adults consists of a basal elastic-muscular layer (elastic fibrils and longitudinally arranged smooth muscle cells [SMCs]) and a more edematous, collagenous (fibrous) luminal layer. The basal layer contained desmin-positive SMCs, whereas the luminal layer usually contained desmin-negative SMCs ( $m A b$ anti-Desmin negative and $\mathrm{mAb}$ anti- $\alpha$-actin positive). Only a few scattered monocytes or macrophages were observed in the thickened intima ( $\mathrm{mAb}$ anti-human macrophage or $\mathrm{mAb}$ Leu-M3 positive). Endothelial cells were variably present in our cross sections. These observations agree with earlier studies $(30,31)$.

After perfusion with anticoagulated blood and staining with the biotinylated platelet mAb $6.74 \alpha$-Ib and the avidinbiotin method, platelets and platelet thrombi on coronary cross sections were studied by light microscopy. There was no platelet material observed without perfusion. On cross sections of healthy, adult coronary arteries, platelet deposition was mainly seen on the luminal side of the intima as a single row of elongated aggregates of small (maximum diameter $<10 \mu \mathrm{m}$ ) to medium size (maximum diameter between 10 and $30 \mu \mathrm{m}$ ) and on the adventitia as large spherical aggregates (maximum diameter between 30 and $100 \mu \mathrm{m}$; Fig. $1 \mathrm{~A}$ ). Platelet deposition on the remaining part of the intima and on the media was minimal and consisted of single platelets or very small and loose aggregates. The basal elastic-muscular layer of the intima had the same reactivity as the media, whereas the luminal fibrous layer was sometimes slightly more reactive. The relative lack of activity in the media was not caused by the use of cross sections, since longitudinal sections of the media gave the same results.

We removed the adventitia to explore whether initial (upstream) exposure to very reactive adventitia does affect platelet interaction with the inner layers. No differences in platelet deposition on media and intima were observed between cross sections with and without adventitia. Some cross sections showed an effect of blood flow direction on the luminal side of the intima. Upstream thrombi on the luminal side of the intima were larger than downstream thrombi on the luminal side of the intima. This phenomenon was probably caused by platelets that collide with the vessel wall.

Cross sections of coronary arteries from newborns showed the same pattern of platelet deposition as the adult specimens
(Fig. $1 B$ ). The surrounding tissue of the artery was also very reactive, whereas the media was sometimes somewhat more reactive than the media in adult coronary arteries.

Umbilical cord arteries. Umbilical cord arteries are "small" elastic arteries without atherosclerosis. In contrast to coronary arteries, they have no clear intima and media; an internal elastic lamina is absent. Umbilical cord arteries showed the same adhesion pattern as the normal coronary arteries after perfusion with anticoagulated blood. Platelet deposition was mainly on the luminal side and on the adventitia (Fig. 1C).

Atherosclerotic coronary arteries. Atherosclerotic plaques had very diverse forms. Some of them consisted completely of dense connective tissue, or of loose connective tissue, whereas in other atherosclerotic plaques dense connective tissue alternated with loose connective tissue. Some plaques had more complex morphologic features, which sometimes indicated the presence of an organized thrombus. The typical atheromatous plaque, consisting of a basocentral atheroma and a thin fibrous cap, was not often seen in the coronary arteries of patients in this study.

In atherosclerotic plaques an increase in SMCs, monocytes, macrophages, and dense connective tissue was observed. Macrophages were often transformed into "lipid-laden" foam cells. T lymphocytes (mAb Leu-4) were also found. In spite of the great variation in cell types and distribution of cells, the various cell types were often arranged in layers or groups.

After perfusion, most of the atherosclerotic intima was covered by platelet aggregates. Larger and more compact aggregates were mainly found in the upper half of the intima (Figs. 2 and 3 ). The size of the aggregates could reach that found on the adventitia and be similar to that on the adventitia of normal coronary arteries. The basal layer of the intima was less reactive. A relationship between the reactivity of the plaque towards platelets and a special cell type in the plaque was not observed. Platelet deposition was restricted to the connective tissue, since areas with high cell numbers and hardly any connective tissue (cell infiltrates, areas with many foam cells) were less reactive. In patients with eccentric plaques, the opposite part of the vessel wall served as an internal control. The normal-appearing wall opposite to an eccentric plaque showed the same pattern of platelet deposition as the healthy coronary artery. Although we have not systematically studied a possible effect of patient age, we have no indications that there was one.

In advanced plaques, no platelet deposition was observed on lipid-filled areas, in contrast with the fibrous cap and the shoulder regions (cellular sides) of the plaque (Fig. $4, A$ and $B$ ). Collagenous layers were usually very reactive, although a gradation could be seen: superficial collagenous layers of the plaque were more reactive than deeper collagenous layers of the plaque (Fig. 3). The media and the elastic-muscular layer of the intima of coronary arteries with atherosclerotic plaques were always less reactive towards platelets than the remaining part of the intima. In an occasional section the strictly circular

Figure 5. SEM micrographs. (a) Platelet deposition on the normal intima ( $i)$. Platelet aggregates are shown on the luminal side of the intima $(*)$. Some single (dendritic) platelets are seen on the underlying intima (arrows). $l$, lumen. Bar $=10 \mu \mathrm{m}$. ( $b$ ) Platelet deposition on the media $(M)$. Platelets adhere predominantly to the connective tissue between the cells (arrows). Bar $=10 \mu \mathrm{m}$. (c) Platelet deposition on the adventitia $(A)$. A platelet aggregate is shown on thick strands of collagen. Bar $=5 \mu \mathrm{m} .(d)$ Platelet deposition on an atherosclerotic intima $(i)$. Platelet aggregates on the fibrous cap of an atherosclerotic plaque. $l$, lumen. Bar $=10 \mu \mathrm{m}$. (e) Platelet deposition on an atherosclerotic intima. Platelet aggregates on thick strands of collagen. Platelets that adhere directly on collagen strands are clearly spread (arrows). Bar $=5 \mu \mathrm{m}$. 
Table II. Platelet Coverage on Cross Sections at Low Magnification

\begin{tabular}{lccc}
\hline $\begin{array}{c}\text { Coronary } \\
\text { segment* }\end{array}$ & $\begin{array}{c}\text { No. cross } \\
\text { sections } \\
\text { evaluated }\end{array}$ & $\begin{array}{c}\text { Platelet coverage } \\
\text { intima } \pm \text { SEM }\end{array}$ & $\begin{array}{c}\text { Platelet coverage } \\
\text { media } \pm \text { SEM }\end{array}$ \\
\hline & & $\%$ & $\%$ \\
RCA atherosclerotic & 5 & $30.7 \pm 2.0$ & $10.3 \pm 2.2$ \\
LCX atherosclerotic & 5 & $26.7 \pm 3.9$ & nd \\
LAD atherosclerotic & 3 & $19.3 \pm 1.7$ & $4.5 \pm 1.4$ \\
LCX normal & 4 & $14.1 \pm 1.9$ & $3.2 \pm 1.2$ \\
LAD normal & 5 & $10.7 \pm 1.4$ & $1.5 \pm 0.3$ \\
\end{tabular}

ND, not determined. RCA, right coronary artery; LCX, left circumflex artery; LAD, left anterior descending artery. * Segments are all from the same patient and are perfused with blood from the same donor.

organization of the media had disappeared locally and the content of connective tissue was increased. Such a media was more reactive towards platelets than an unaffected one.

We reversed the orientation of cross sections in the perfusion chamber to see whether platelet deposition to various layers of eccentric atherosclerotic plaques was influenced by the blood flow direction. We did not observe differences in the pattern of platelet deposition when we altered the orientation of cross sections.

\section{Scanning electron microscopic studies}

With the use of SEM techniques, platelet deposition could be observed without the use of a platelet antibody. Fig. 5, $a-c$, showed platelet deposition on the intima, the media, and the adventitia of a normal coronary cross section, respectively. Fig. $5 d$ shows platelet aggregates on the luminal side of an atherosclerotic cross section (same artery as shown in Fig. 4).

These electron micrographs clearly show that the structure of the various artery layers was basically different. In the healthy intima and in the media, cells and finely dispersed collagen were present, whereas in the adventitia, the collagen fibrils were bundled to form thick strands. In some areas of the atherosclerotic plaque, the collagen fibrils were organized in the same way as in the adventitia (Fig. $5 e$ ).

In the healthy intima, platelet aggregates were observed on the luminal side. Some single (dendritic) platelets were seen on the underlying intima (Fig. $5 a$, arrows). Platelets that adhered on the media adhered predominantly to the connective tissue between the cells (Fig. $5 \mathrm{~b}$ ). The aggregates on the plaque con- sisted of several layers of platelets (Fig. $5 e$ ). Although they appeared to be equally large, they were less compact than the aggregates on the adventitia (Fig. $5 c$ ) and the platelets were more spread.

\section{Quantitative evaluation with the IBAS image analysis system.}

Our study on the reactivity of the normal vessel wall and atherosclerotic plaques relies on qualitative evaluation because of the complex nature of the plaque. To obtain some quantitative insight, a limited morphometric evaluation was performed. Table II shows the platelet coverage of cross sections derived from atherosclerotic and normal coronary segments from one patient. The evaluated cross sections were tested in two or three different perfusion runs, but with blood of a single donor. The atherosclerotic intima had mean platelet coverages ranging between 19.3 and $30.7 \%$, whereas the normal intima had significantly lower coverages $(<14.1 \% ; P<0.001)$. The platelet coverages of the media were between 1.5 and $10.3 \%$ (mean values) and were significantly lower than the platelet coverages of the intima $(P<0.005)$. Thrombi on the (atherosclerotic) vessel wall were classified in four groups: $<10 \mu \mathrm{m}^{2}$ (single platelets and small aggregates), and $<100,<500$, and $\geq 500 \mu \mathrm{m}^{2}$.

In Table III we present results of three locations in an artery segment with a concentric atherosclerotic plaque, one location on the atherosclerotic plaque with a high degree of coverage (ath. pl. A), one location on the atherosclerotic plaque with a low degree of coverage (ath. pl. B), and one location on the media. Three cross sections per coronary segment were evaluated. Table IV shows the data of a coronary segment with an eccentric atherosclerotic plaque. Instead of three cross sections, only one (representative) cross section was evaluated. Per location (plaque or opposite wall of the plaque) six adjacent fields were evaluated and averaged $( \pm \mathrm{SD})$.

It is evident from Tables III and IV that the atherosclerotic plaque had more large aggregates $\left(\geq 100 \mu \mathrm{m}^{2}\right)$ than the media or the "healthy" opposite wall of the eccentric plaque $(P$ $<0.01)$. The total number of platelets and platelet thrombi and the total platelet coverage in the atherosclerotic plaque were increased compared to media values $(P<0.05$ and $<0.005)$. Increased platelet coverage in the intima was not accompanied by an increased number of platelets or platelet aggregates, but by an increased percentage of large aggregates.

\section{Immunohistochemical studies}

As described above, we found strongly increased platelet deposition on well-defined areas of atherosclerotic plaques. Immunohistochemical staining of adhesive proteins was performed in

Table III. Platelet Thrombi: Atherosclerotic Plaque vs. Media

\begin{tabular}{lrrrr}
\hline & \multicolumn{3}{c}{ Areas \pm SEM } \\
\cline { 2 - 5 } Location & $<10 \mu \mathrm{m}^{2}$ & $<100 \mu \mathrm{m}^{2}$ & $<500 \mu \mathrm{m}^{2}$ & $2500 \mu \mathrm{m}^{2}$ \\
\hline & & & & \\
& & & & \\
Tath. pl. A & $46.9 \pm 5.1$ & $29.6 \pm 4.0$ & $19.6 \pm 0.7$ & $3.9 \pm 1.3$ \\
Media & $73.7 \pm 4.6$ & $24.5 \pm 4.0$ & $1.8 \pm 0.9$ & 0 \\
ath. pl. B & $41.6 \pm 3.7$ & $43.0 \pm 1.3$ & $13.6 \pm 2.3$ & $1.7 \pm 0.8$
\end{tabular}

ath. pl., atherosclerotic plaque. Two different locations in the concentric plaque are evaluated. Location A has a significantly higher platelet coverage $(33.3 \pm 1.3 \%)$ than location B $(22.0 \pm 2.8 \%)$. 
Table IV. Platelet Thrombi: Atherosclerotic Plaque vs. Normal Intima

\begin{tabular}{|c|c|c|c|c|c|}
\hline \multirow[b]{2}{*}{ Location } & \multicolumn{4}{|c|}{ Areas \pm SD } & \multirow[b]{2}{*}{ Total platelet coverage $\pm \mathrm{SD}$} \\
\hline & $<10 \mu \mathrm{m}^{2}$ & $<100 \mu \mathrm{m}^{2}$ & $<500 \mu \mathrm{m}^{2}$ & $\geq 500 \mu \mathrm{m}^{2}$ & \\
\hline & \multicolumn{4}{|c|}{$\%$} & $\%$ \\
\hline atherosclerotic pl. & $7.9 \pm 3.1$ & $24.5 \pm 9.0$ & $40.3 \pm 10.1$ & $27.3 \pm 9.8$ & $45.7 \pm 2.3$ \\
\hline "Normal" intima" & $14.5 \pm 3.3$ & $59.4 \pm 11.7$ & $25.7 \pm 11.8$ & $0.4 \pm 0.6$ & $5.7 \pm 2.4$ \\
\hline
\end{tabular}

atherosclerotic pl., atherosclerotic plaque; SD, standard deviation. A perfused cross section of this coronary segment is shown in Fig. 2 . ${ }^{*}$ The "normal" intima (opposite wall of the atherosclerotic plaque) includes the aggregates on the luminal side of the intima.

order to find which protein could be responsible for this increased thrombogenicity. For proteins that showed increased staining, we compared the localization with the areas of increased platelet deposition (Fig. 6).

vWF was present in endothelial cells and in the extracellular matrix immediately beneath the endothelial cells. No vWF was detected in other cells or matrix structures neither in the normal vessel wall nor in the atherosclerotic vessel wall.

Laminin and collagen type IV were diffusely distributed in the normal and atherosclerotic intima. The immunostaining was more intense on the luminal side of the intima (and on the luminal side of small blood vessels in the adventitia), indicat-
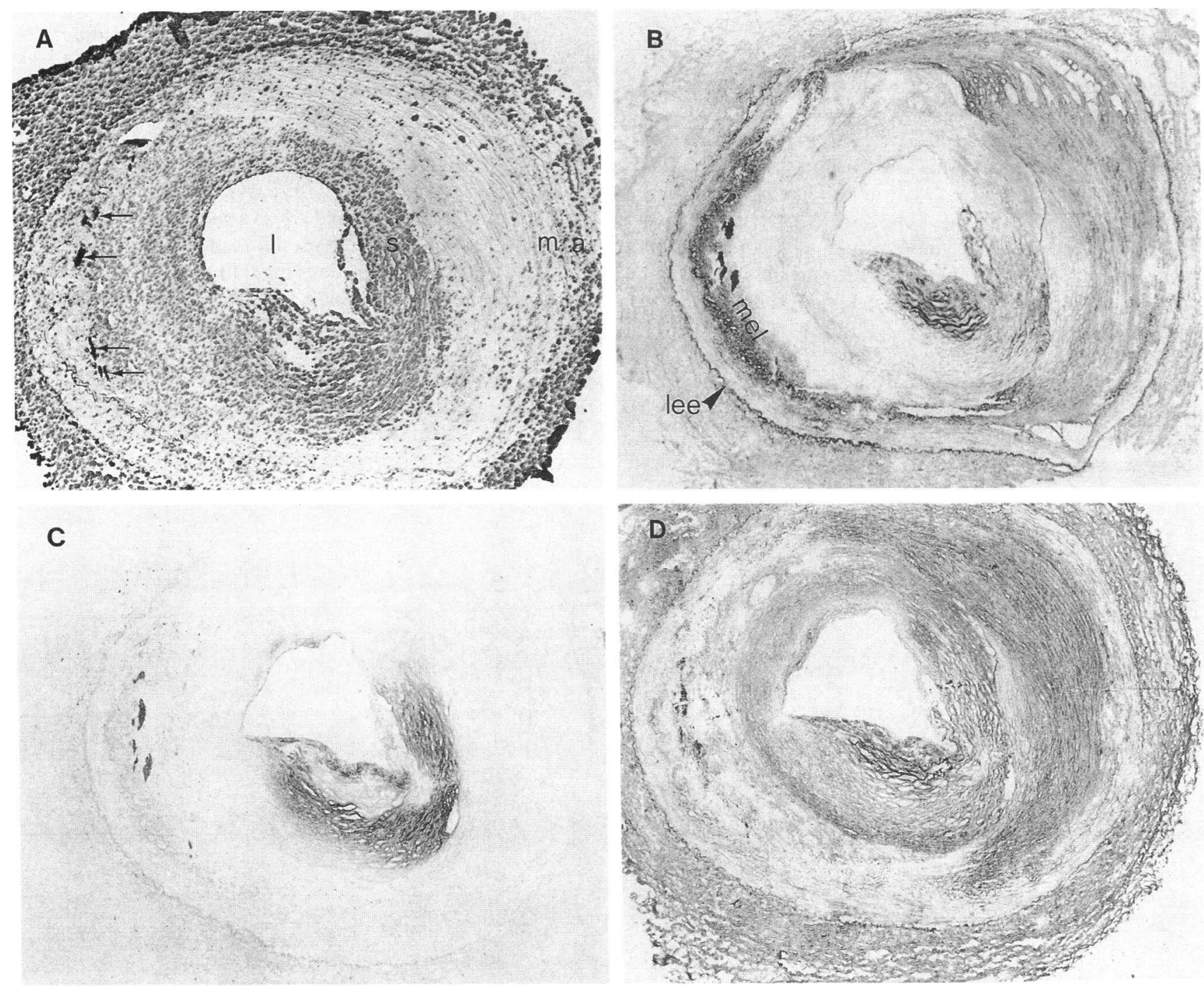

Figure 6. Four cross sections of a coronary artery with a concentric atherosclerotic plaque in which the superficial part $(s)$ of the plaque is more reactive towards platelets than the deeper part of the plaque. $(A)$ Cross section after perfusion with blood and staining with an anti-Gp Ib antibody; $(B-D)$ cross sections without perfusion, but stained with antibodies against vitronectin $(B)$, thrombospondin $(C)$, and collagen type I $(D)$. $l$, lumen; $m$, media; $a$, adventitia; lee, lamina elastica externa; $M E L$, myo-elastic layer; $\rightarrow$, calcium deposits. 


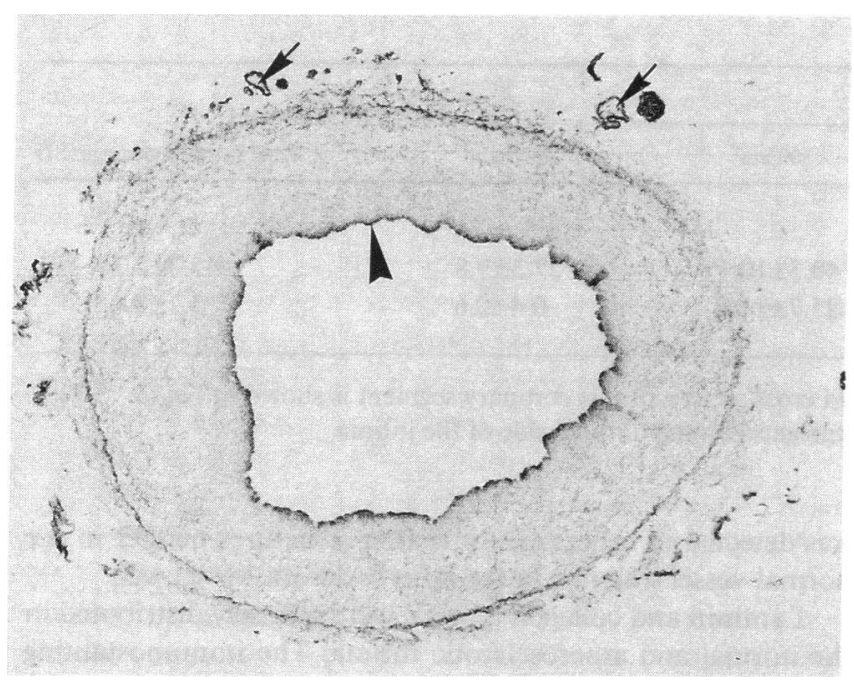

Figure 7. Cross section of a healthy coronary artery stained with an antibody against collagen type IV. Most intensive staining is visible on the luminal side of the intima (arrowhead) and in small vessels in the adventitia (arrows).

ing that laminin and collagen type IV were mainly present in the endothelial basement membrane (Fig. 7).

Vitronectin and fibronectin were diffusely distributed in the matrix of the normal intima. Fibronectin showed a fibrillar staining pattern, whereas the vitronectin pattern was more granular. In contrast to fibronectin, vitronectin was clearly present on the internal elastic lamina. In some plaques a more intense staining pattern of both proteins was observed. Some areas, particularly necrotic areas, stained more intensely (Fig. 8).

The normal intima showed no diffuse staining for thrombospondin, in contrast to vitronectin and fibronectin. A luminal lining was often observed in the normal intima, although this line was not continuous. The deeper part of the normal intima was negative (Fig. 9). Clearly defined areas in athero- sclerotic plaques stained strongly positive. Some of the border lines corresponded with platelet reactive areas, but, as shown in Fig. 6, the platelet-reactive area could also exceed these border lines.

Collagen types I and III showed an identical distribution both in the normal and in atherosclerotic vessel wall. Intense staining was often seen in superficial layers of the plaque. Collagen type III was sometimes enriched on the luminal side of the normal intima (Fig. 10). Platelet-reactive areas were always intensely stained for collagen types I and III, but there were also intensely stained areas that were not reactive towards platelets (Fig. 6). Collagen type V showed a more or less comparable distribution as collagen types I and III, but the pattern was less well defined.

Collagen type VI showed a completely different staining pattern compared with the collagen types already mentioned. Some areas stained clearly more intensely both in the normal intima and in the atherosclerotic plaque. More intensely stained areas in the atherosclerotic plaque were irregularly distributed.

The distribution of fibrinogen in the arterial wall was variable. An increased staining for fibrinogen was often seen, but normal looking intima could also stain positively without being reactive towards blood platelets. We did not observe differences in staining patterns between post-mortem and fresh coronary arteries.

\section{Inhibition studies}

$v W F$. Addition of the mAbs CLB-RAG35 (against the platelet binding domain on vWF) and AK2 (against the vWF binding site of $\mathrm{GpIb}$ ) to the perfusates resulted in an almost complete inhibition of platelet deposition (Fig. 11). VWF-free blood was not always sufficient to inhibit platelet adhesion completely, but addition of CLB-RAG35 resulted in a complete inhibition.

Fibronectin. For studies in which the role of fibronectin was studied we used citrated blood, since we recently observed that (un)fractionated heparin inhibited platelet adhesion to purified fibronectin. Preincubation of cross sections with an antifibronectin $\mathrm{Ab}$, perfusions with fibronectin-free blood, or

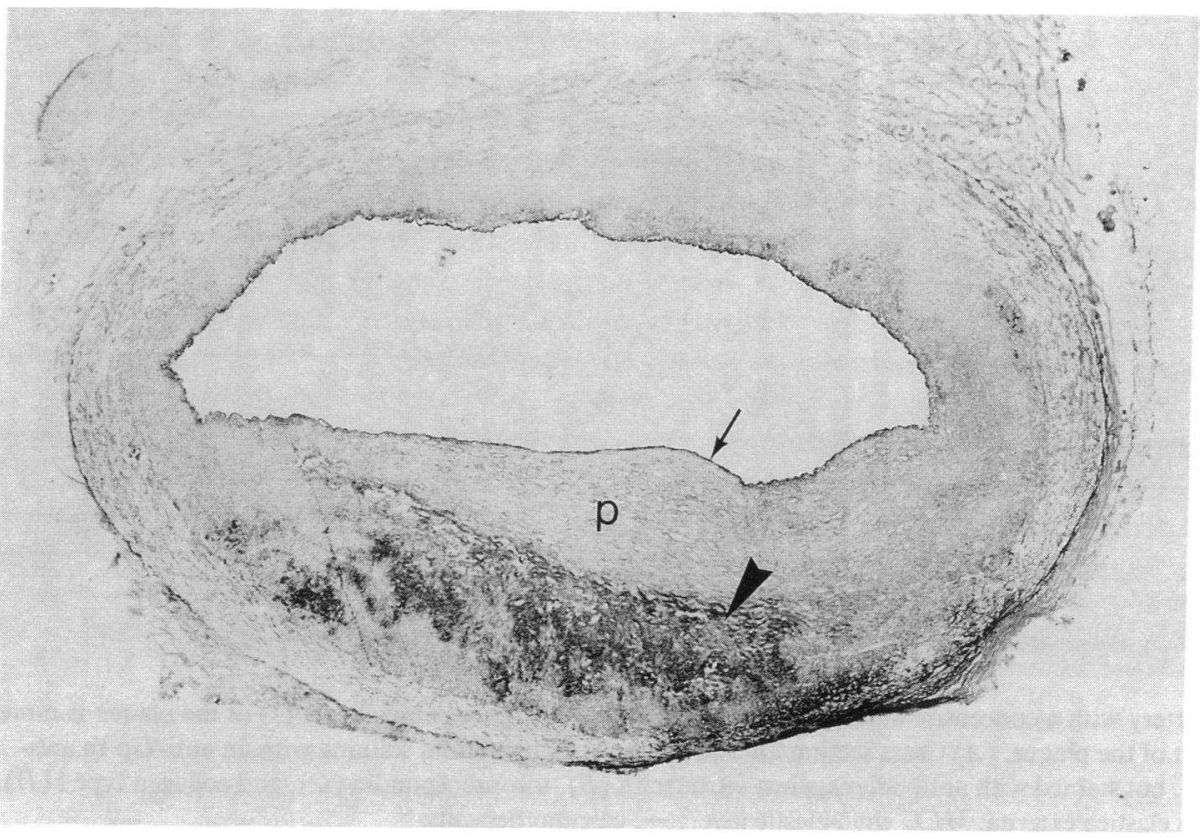

Figure 8 . Cross section of a coronary artery with an eccentric plaque $(p)$, stained with an antibody against vitronectin. Staining is most intensive on the luminal side of the vessel wall (arrow), and in the necrotic area of the plaque (arrowhead). 


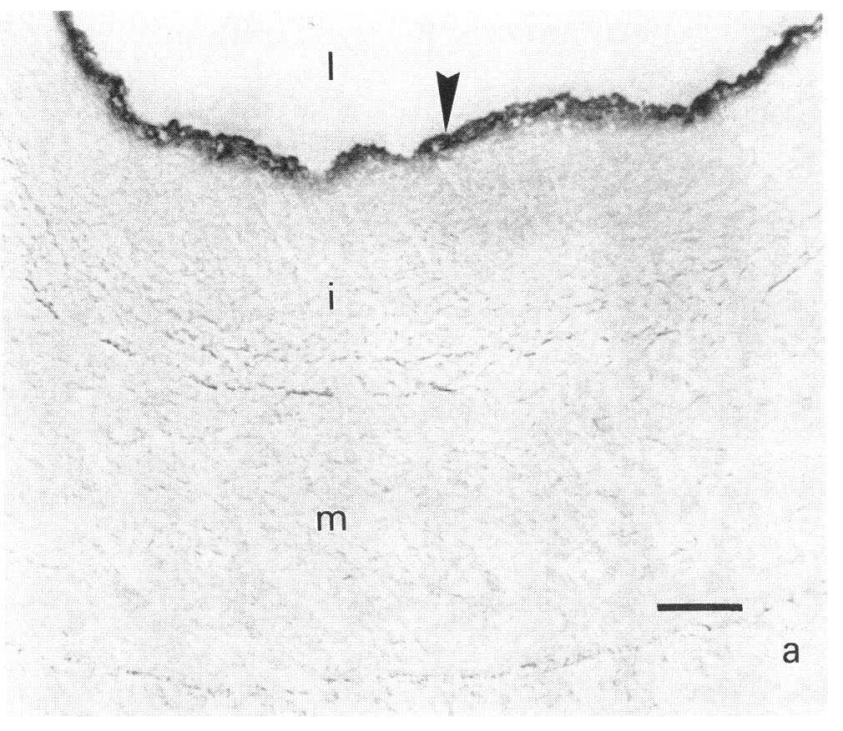

Figure 9. Section of a normal coronary artery stained with an antibody against thrombospondin. Only the luminal side of the intima ( $i$ ) reacts positive (arrowhead). $l$, lumen; $m$, media; $a$, adventitia. Bar $=50 \mu \mathrm{m}$.

with a combination of these two conditions did not result in a decrease in aggregate size. Perfusions with fibronectin-free blood sometimes resulted in less compact aggregates, but the en face platelet coverage was not decreased. To compare these results with earlier studies on endothelial cell matrix (ECM) (20), we performed parallel experiments on cross sections of coronary arteries and ECM both at low (shear rate $=400 / \mathrm{s}$ ) and high shear rates (shear rate $=1,600 / \mathrm{s}$ ). Table $\mathrm{V}$ shows that fibronectin had no significant effect on platelet deposition on atherosclerotic plaque in contrast to platelet deposition on ECM, which was significantly decreased.

Fibrinogen/fibrin. The role of fibrinogen/fibrin in adhesion to the atherosclerotic plaque was studied via inhibition of its receptor. Gp IIb-IIIa acts as primary receptor for adhesion to fibrinogen (32). Platelet adhesion to fibrinogen or fibrin was completely inhibited by the synthetic peptide dRGDW. dRGDW $(100 \mu \mathrm{M})$ completely blocked aggregate formation on cross sections of coronary arteries, but had no effect on platelet adhesion (Fig. 12), ruling out an essential role of fibrinogen.

Laminin. VLA-6 (Gp Ic-IIa) is the receptor for laminin involved in adhesion in flow. Concentrations of $\mathrm{mAb} \mathrm{GoH} 3$ against (VLA-6), very late antigen-6 which completely blocked platelet adhesion on purified laminin, had no effect on platelet deposition on the atherosclerotic plaque.

Thrombospondin. Studies with isolated thrombospondin coated on a glass coverslip have shown that thrombospondin causes extensive platelet adhesion up to a shear rate of $1,600 / \mathrm{s}$ (33). Thrombospondin contains at least 12 calcium binding sites, and thrombospondin in a calcium-depleted form does not support platelet adhesion $(33,34)$. If the increased amount of thrombospondin in the atherosclerotic plaque was responsible for the increased platelet deposition, perfusions with citrate or preincubation of the atherosclerotic plaque with EDTA should abolish the increased platelet deposition. Perfusion studies in which citrated blood was compared with blood anticoagulated with LMWH showed no difference. Pretreatment of tissue sections with EDTA also had no effect on platelet deposition (not shown).

Collagens. Atherosclerotic lesions were enriched in collagens I, III, V, and VI. Since no polyclonal or monoclonal Abs directed against different collagens inhibiting adhesion were available, we had to make use of previous results obtained on isolated proteins. These studies showed that no adhesion occurred to collagen type $\mathrm{V}$ and that adhesion to collagen type $\mathrm{VI}$ was low at $1,600 / \mathrm{s}(35)$, the shear rate used for the platelet adhesion studies on atherosclerotic plaques. We therefore concentrated our studies on collagen types I and III. Antibody studies with Abs against $\mathrm{Gp}$ Ia and perfusion studies with blood of a patient whose platelets lacked $80 \%$ of Gp Ia had demonstrated that Gp Ia-IIa was the most important platelet receptor
Figure 10. Cross section of a coronary artery with an eccentric plaque stained with an antibody against collagen type III. Intense staining is shown in the adventitia, the plaque $(*)$, and on the healthy side of the vessel wall (arrowhead). 

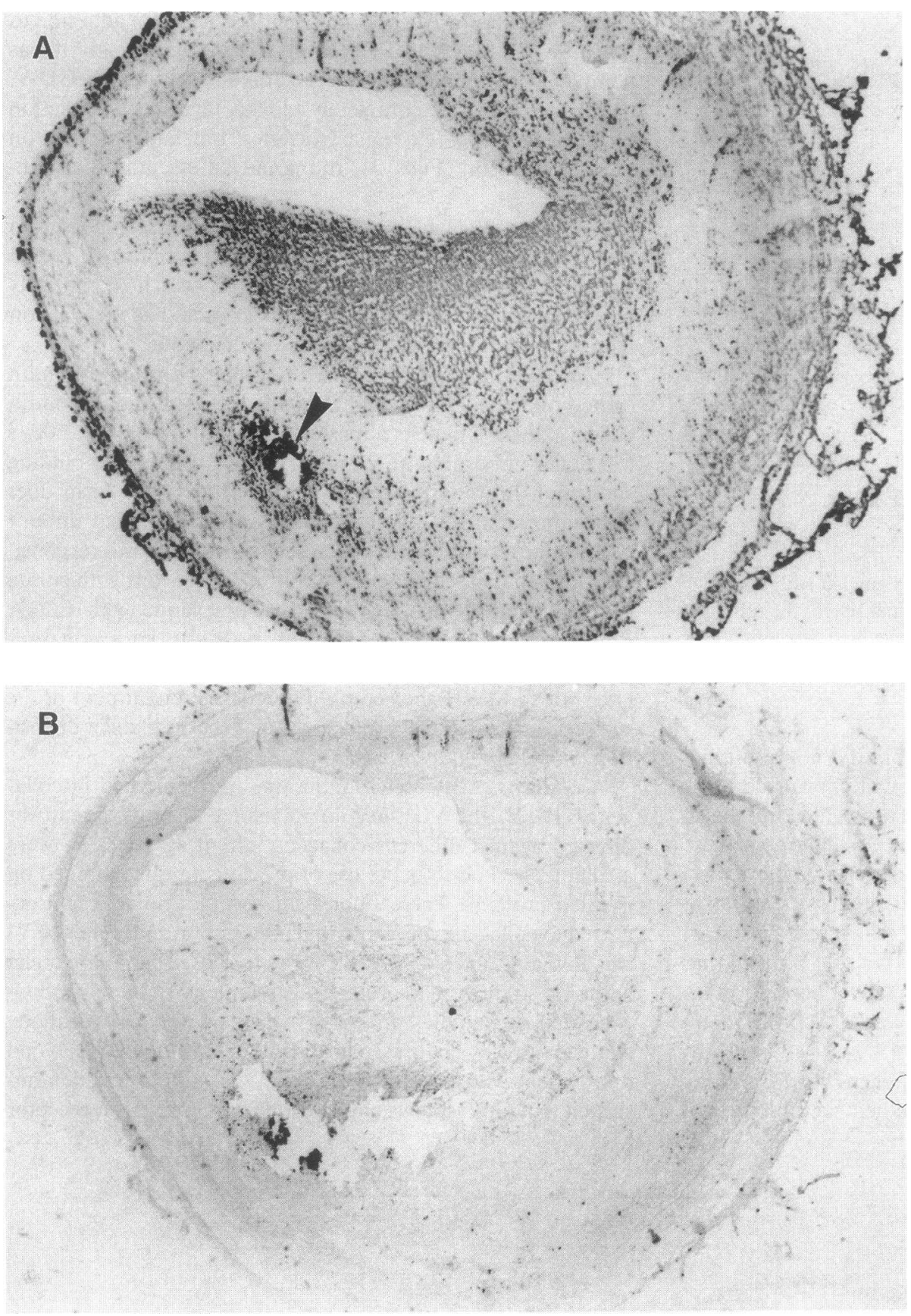

Figure 11. Perfusions over cross sections of atherosclerotic coronary arteries. $(A)$ Control; $(B)$ mAb CLBRAG35 (against the platelet binding domain on vWF) added to the perfusate resulted in an almost complete inhibition of platelet deposition. Calcium deposits were visible in the necrotic, lipid-rich, deeper part of the plaque (arrowhead). for collagen (28). The platelet deposition on atherosclerotic plaques was therefore studied with blood of our patient with Gp Ia deficiency. The results are visible in Fig. 13. A pronounced decrease in aggregate size was observed.

A novel leech protein ( $r$-LAPP) inhibiting platelet aggregation with collagen, expressed in yeast (26), was recently studied in our flow system. It was a potent inhibitor of adhesion to collagen types I, III, and IV, but not to collagen type VI. This recombinant protein was also a potent inhibitor of thrombus formation on the atherosclerotic plaque (Fig. 13).

\section{Discussion}

In this study, semi-thin cross sections of coronary arteries were exposed to flowing blood in a rectangular perfusion chamber. With this method it is possible to investigate the platelet inter- action with deeper layers of the (atherosclerotic) vessel wall. This study is the first in which platelet deposition on all (relative intact) layers of the vessel wall is investigated under the same standardized shear conditions. The method used has the advantage that the reactivity of the plaque by itself is studied without other interfering factors in the bloodstream, and it opens ways for the study of new therapeutic approaches.

We have demonstrated that atherosclerotic vessel walls were more reactive towards platelets than normal vessel walls. The increased platelet deposition was limited to specific parts of the atherosclerotic plaque. Superficial layers of a plaque were often more reactive towards platelets than deeper layers. Some deeper layers of the atherosclerotic plaque could even be less reactive than the normal intima. Atherosclerotic plaques were also different among themselves.

The variability within and between plaques may explain 
Table V. Platelet Adhesion on the Endothelial Cell Matrix (ECM) vs. Platelet Deposition on the Atherosclerotic Plaque (ath.pl.) in Relation to Fibronectin

\begin{tabular}{|c|c|c|c|c|}
\hline \multirow[b]{2}{*}{ Surface } & \multirow[b]{2}{*}{ Surface preincubation } & \multirow[b]{2}{*}{ Perfusate } & \multicolumn{2}{|c|}{$\begin{array}{l}\text { Platelet adhesion/deposition } \\
\text { (surface coverage; mean } \pm \text { SD) }\end{array}$} \\
\hline & & & Sr $400 / \mathrm{s}$ & Sr $1,600 / \mathrm{s}$ \\
\hline & & & \multicolumn{2}{|c|}{$\%$} \\
\hline ECM & Control & Whole blood & $24.4 \pm 1.7$ & $56.1 \pm 0.1$ \\
\hline ECM & Control & reconst. normal pl & ND & ND \\
\hline ECM & $\mathrm{F}\left(\mathrm{ab}^{\prime}\right)_{2}$ anti-FN & reconst. $\mathrm{FN}^{-} \mathrm{pl}$ & $8.9 \pm 1.4^{*}$ & $13.4 \pm 2.6^{*}$ \\
\hline ath.pl. & Control & Whole blood & $21.0 \pm 2.5$ & $29.3 \pm 0.9$ \\
\hline ath.pl. & Control & reconst. normal pl & $18.3 \pm 1.1$ & $31.1 \pm 3.1$ \\
\hline ath.pl. & $\mathrm{F}\left(\mathrm{ab}^{\prime}\right)_{2}$ anti-FN & reconst. $\mathrm{FN}^{-} \mathrm{pl}$ & $22.7 \pm 1.7^{\mathrm{ns}}$ & $31.1 \pm 1.7^{\mathrm{ns}}$ \\
\hline
\end{tabular}

Surfaces were preincubated with $\mathrm{F}\left(\mathrm{ab}^{\prime}\right)_{2}$ fragment of the anti-fibronectin (FN) antibody. As a control surfaces were preincubated with buffer. Perfusions were performed with citrated whole blood, platelets, and red blood cells reconstituted (reconst.) with either normal plasma (pl.) or fibronectin-free plasma $\left(\mathrm{FN}^{-} \mathrm{pl}\right)$. Four coverslips per condition were evaluated. NS, not significant different from control ( $t$ test). ND, not determined. ${ }^{*} P<0.005$.

why others did not find increased reactivity of the atherosclerotic plaque. Locally distinct concentrations of adhesive or thrombogenic components can cause different reactivity of various parts of an atherosclerotic plaque. For these reasons, work with whole tissue extracts is unlikely to be valuable.

We have demonstrated that platelet adhesion to subendothelium, but particularly to adventitia and atherosclerotic plaques, leads to large aggregate formation, whereas adhesion to normal intima and media in general does not cause this. It is not easy to distinguish the initial adhesion events from subsequent platelet-platelet interactions, because it is not possible to inhibit aggregation completely without also affecting platelet adhesion at the shear rate we have used. To obtain some insight, we studied platelet adhesion after 1-min perfusion (results not shown). Most of the platelets adhered at sites where later on platelet aggregates were formed. This suggests that regions of the plaque that cause maximal activation of adhered platelets, leading to aggregate formation, are also those that cause the most pronounced initial adhesion.

We observed no difference in the pattern of platelet deposition between blood anticoagulated with citrate and blood anticoagulated with LMWH and desulphato-hirudin, although in vitro work of the collagen receptor shows a distinct dependence on divalent cations (36). However, we have analyzed the aggregate formation with en face evaluation, which does not permit the analysis of small differences in thrombus height and density. Our results are in line with previous work on adhesion to subendothelium $(37,38)$. Platelet adhesion to a more complex matrix is evidently not sensitive to low calcium/magnesium concentrations, in contrast with adhesion to purified proteins. It is plausible that platelet adhesion to a more complex matrix is less dependent on calcium/magnesium, because it involves a series of different parallel and consecutive receptorligand interactions.

Exposure of semi-thin cross sections of coronary arteries to flowing blood facilitates the study of the thrombogenicity of the atherosclerotic plaque, but the great variation in plaque composition, even in serial cross sections, makes this study complex. This is one of the reasons why we opted for a qualitative evaluation. To obtain some idea of aggregate dimensions and platelet coverage, however, a series of cross sections was evalu- ated with a computerized image analysis system (IBAS). These data confirm the highly significant increase in platelet coverage and platelet aggregate dimensions on the atherosclerotic plaque compared with the media and the normal intima. The computerized image analysis system as used here may have some inherent problems. Platelet coverages determined at low magnification are probably somewhat overestimated (Table II), but intima and media values of the different coronary segments are comparable. Less reactive layers, like the media and the normal intima, show decreased platelet staining and are easily overestimated. Nevertheless a clear difference between atherosclerotic and normal intima is demonstrated. Platelet coverages determined at higher magnification are more realistic ( $\mathrm{Ta}-$ bles III and IV). Data about aggregate dimensions show that an increased platelet coverage in the atherosclerotic plaque is accompanied by an increased percentage of large aggregates. These quantitative results agree very well with the qualitative description.

Under the conditions in this study, the cholesterol-rich pool in the atheroclerotic plaque does not promote platelet deposition, although it is conceivable that atherosclerotic plaques with a pool of extracellular lipid in the intima lead more frequently to plaque rupture and subsequent thrombus formation in vivo $(39,40)$. The presence of tissue factor will increase the thrombogenicity of the atherosclerotic plaque dramatically, and recent studies (41) show that thrombin inhibitors can block the development of acute platelet thrombus in "an in vivo pig model." Despite this knowledge, it remains unclear which components or factors in the atherosclerotic vessel wall are responsible for increased thrombogenicity and how they work together with factors from outside the vessel wall. Although "in vivo experiments" with animal models have produced valuable results $(42,43)$, it is not clear that these results can be extrapolated to the human system. For example, Badimon and Badimon (42) found that the media from the vessel wall of the pig was considerably more reactive towards platelets. We found similar increased reactivity of the media in cross sections of rabbit arteries (our unpublished results). Differential distribution of adhesive proteins in different species or even in different arteries within a species (44) can result in altered reactivity of the arteries. Air drying of cross sections could be 

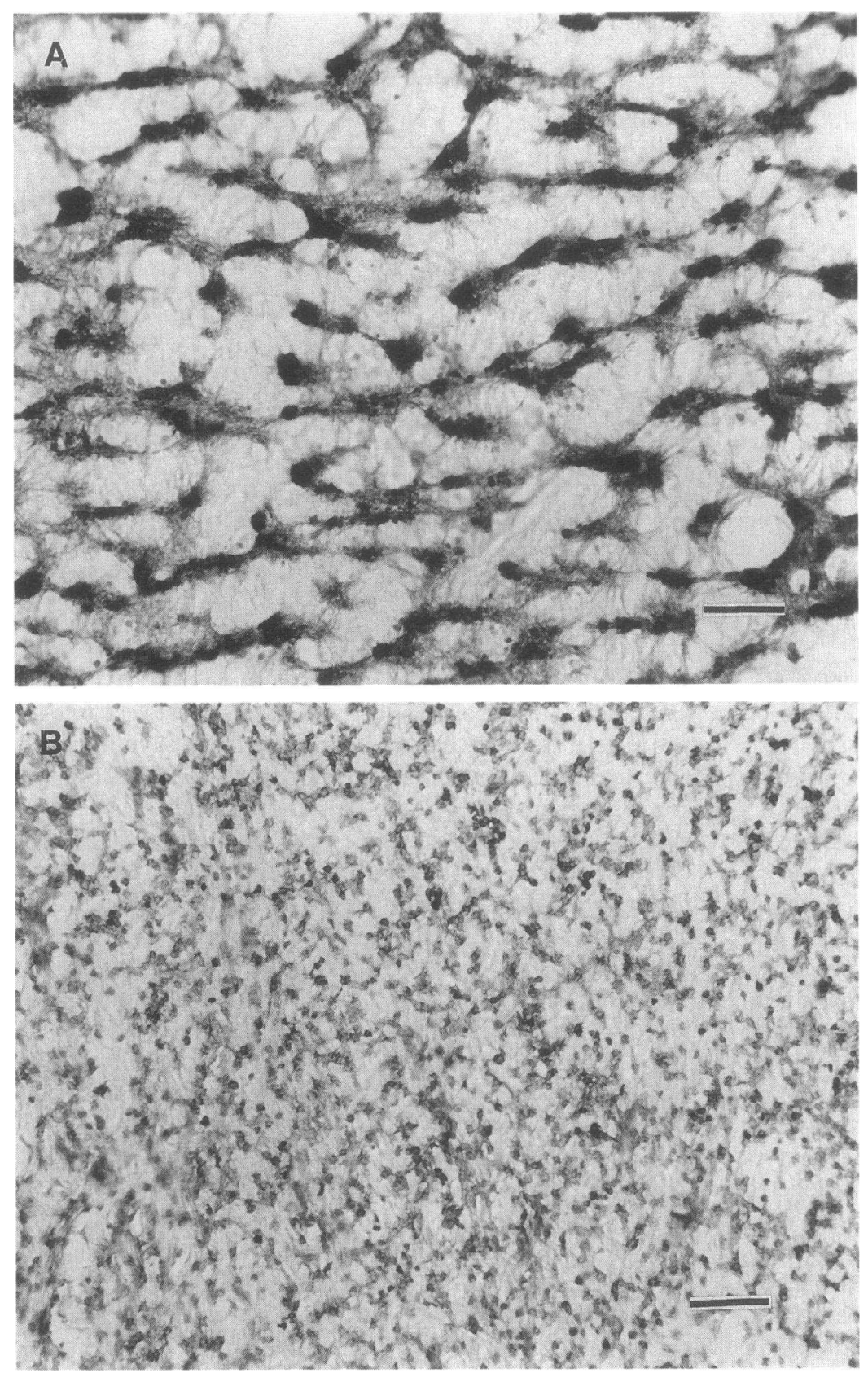

Figure 12. Perfusions over cross sections of atherosclerotic coronary arteries. $(A)$ Control with large aggregates; $(B) 100 \mu \mathrm{M}$ dRGDW peptide added to the perfusate. Platelet aggregate formation was completely abolished in $B$. Bar $=50 \mu \mathrm{m}$. detrimental to surface proteins. However, we have experience with purified fibronectin, vWF, fibrinogen, and various collagen types that were sprayed on glass coverslips. These proteins were also air dried during the spraying process, and gave good platelet adhesion after exposure to flowing blood in the perfusion chamber.

In an attempt to find out why (specific parts of) atherosclerotic plaques in human coronary arteries were more reactive towards platelets, specific Abs against adhesive proteins and platelet receptors were used. As a first step we performed immunohistochemical studies of the various adhesive proteins that may be responsible for this increased reactivity. The localization of vWF, laminin, fibronectin, vitronectin, thrombospondin, fibrinogen/fibrin, and the collagen types I, III, IV, V, and VI was studied and compared with the areas of increased thrombogenicity. Increased presence of collagen types I, III, and $\mathrm{V}$, fibronectin, vitronectin, and thrombospondin has been reported before (45-49). However, these studies concerned mostly atherosclerotic lesions in the aorta. No study was reported in which all adhesive proteins were studied simultaneously in the same atherosclerotic plaques. In this study, we found enrichment of the collagens I, III, and V in large areas of the atherosclerotic plaque. Collagen type VI was also enriched, but the picture was more variable between plaques. Fibronectin has been found to be increased in atherosclerotic plaques (47). Our studies confirmed this, but the staining pattern was rather diffuse. In ELISAs for fibronectin on material obtained from microbiopsies from atherosclerotic plaques, we found up to four times as much fibronectin as in the normal intima (not shown). Vitronectin was also increased in the atherosclerotic plaque, but in contrast to fibronectin the increased staining had a pronounced patchy character. The same staining pattern was 

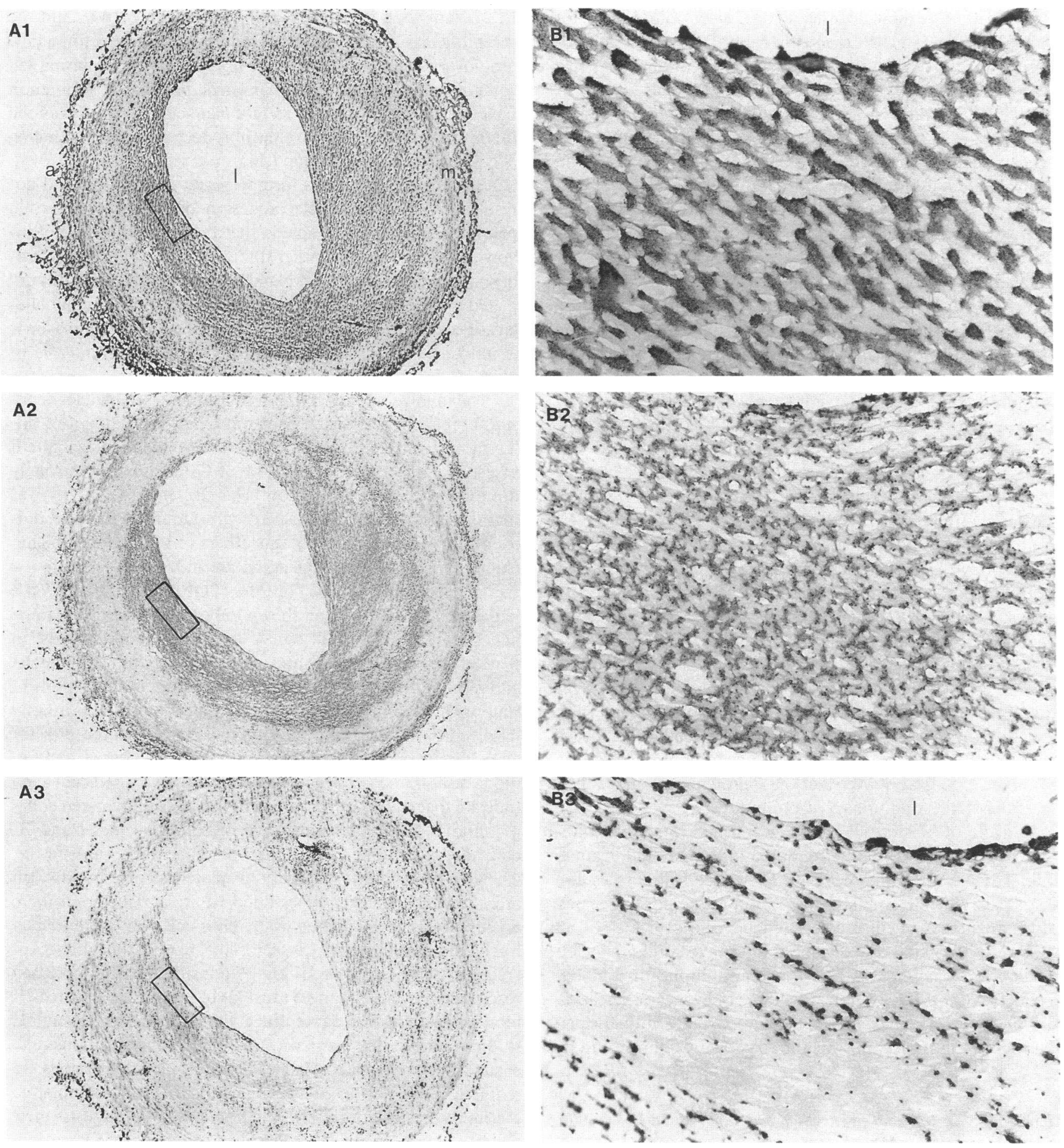

Figure 13. Perfusions over cross sections of an atherosclerotic coronary artery. (1) Control; (2) perfusion with blood of the Gp la-deficient patient; (3) $6 \mu \mathrm{M}$ rLAPP added to the perfusate. The rectangles in $A 1, A 2$, and $A 3$ is magnified 16 times in $B 1, B 2$, and $B 3$, respectively. Platelet coverage of the surface is most decreased in $A 3$. $l$, lumen; $m$, media; $a$, adventitia.

reported by Hayman et al. ( 50 ) for vitronectin. Fibrin has been found to be increased in atherosclerotic coronary arteries by Bini et al. (51). Since we have seen no essential differences in adhesive properties between fibrinogen and fibrin (52), we used an antifibrinogen $\mathrm{Ab}$. We found a patchy distribution in the atherosclerotic plaque that varied strongly between different plaques. Fibrinogen was also variably present in the normal intima. Thrombospondin was strongly and specifically in- creased in atherosclerotic lesions, as reported previously (48). In the normal intima, thrombospondin was only present in a single noncontinuous layer near the lumen. The strong staining of the atherosclerotic lesion concerned only parts of the atherosclerotic lesions. This may correspond to regions where recent proliferation of smooth muscle cells has occurred since thrombospondin is increased in the matrix of proliferating SMCs. Thrombospondin binds to collagen type $\mathrm{V}$ and vice versa (53). 
Colocalization of these two ligands has been found in histochemical studies (48). We could not confirm this for the atherosclerotic plaques: collagen type $\mathrm{V}$ was more diffusely distributed than thrombospondin. As expected, vWF was present in endothelial cells and in the direct subendothelium. No vWF was found in deeper layers of the vessel wall in contrast to experimental data in rabbits in which vWF was found on the lamina elastica interna when SMC proliferation had occurred in an injured blood vessel (54). No enrichment was found in atherosclerotic plaques of the basal lamina components laminin and collagen type IV. The various proteins that were enriched in the atherosclerotic lesions are summarized in Table VI.

The staining patterns found by histochemistry were compared with the areas of increased platelet deposition in serial sections. No exact correspondence was found. The best corresponding pattern was observed for collagen types I and III, where two proteins had exactly the same distribution. The area in which increased collagen types I and III were deposited was often somewhat larger than the area with increased platelet deposition. No correspondence at all was found for collagen type IV, laminin, vitronectin, fibrinogen/fibrin, and vWF. Thrombospondin-positive areas were often reactive for platelets, but there was also increased platelet reactivity in areas without thrombospondin. The staining pattern of fibronectin and collagen types VI and V was diffuse and it was therefore difficult to determine whether colocalization was present.

In the second part of our study, we used various antibodies and inhibitors in order to find out which adhesive proteins were involved in the adhesion of platelets to the areas with increased thrombogenicity. Vitronectin and collagen type $V$ were not studied because previous studies from our laboratory and others (55) have shown that these proteins are only adhesive under static conditions, but not in flow.

The role of plasma vWF was established in studies with mAbs against the GPIb-binding domain of vWF and against GPIb. These same antibodies have been used before to establish the role of vWF in platelet adhesion to the normal vessel wall or the matrix of cultured endothelial cells (19).

Fibronectin in the vessel wall was not important for platelet adhesion, since preincubation with an antifibronectin $\mathrm{Ab}$ had no effect. We can not exclude an effect of fibronectin released from platelets and this can not be studied, since no platelets exist that lack fibronectin. The antibody against fibronectin is

Table VI. Proteins Enriched in Atherosclerotic Plaque

\begin{tabular}{lc}
\hline \multicolumn{1}{c}{ Proteins } \\
\hline Collagen I, III, V, VI & + \\
Collagen IV & - \\
von Willebrand Factor & - \\
Fibronectin & + \\
Fibrinogen & + \\
Vitronectin & + \\
Thrombospondin & ++ \\
Laminin & - \\
\hline
\end{tabular}

- , not enriched; + , enriched; ++ , present in the atherosclerotic plaque, but not in deeper layers of normal intima. less potent than the comparable mAb against $\mathrm{vWF}$, and the receptors involved in the role of adhesion to fibronectin in flow are not known yet. Removal of fibronectin from plasma led sometimes to less compact aggregates, but that phenomenon never resulted in decreased en face platelet coverage. From the literature, it is well known that fibronectin has an enhancing effect on platelet aggregation (56).

Blocking the receptors for fibrinogen and laminin did not result in decreased platelet adhesion on the atherosclerotic plaque. We therefore conclude that these proteins are not involved. We know from earlier studies (33) that purified thrombospondin can not support platelet adhesion in its calcium-depleted form. We found no effect of calcium chelation on platelet adhesion to the atherosclerotic plaque, and for that reason it is unlikely that thrombospondin plays an important role in platelet adhesion.

The involvement of collagen in the increased platelet deposition on atherosclerotic lesions was demonstrated by two different sets of experiments. Studies with blood of a patient whose platelets only possess $20 \%$ of GPIa showed a strongly diminished platelet deposition. This decreased reactivity was mainly reflected in a diminished aggregate size. Previous studies of our own group (57) and others (58) have shown that changes in reactivity of collagens are indeed reflected by the size of platelet aggregates. The $20 \%$ of GPIa still present on the patients' platelets has been shown to be able to support platelet adhesion to some extent (28). A very pronounced decrease in platelet deposition was found with the recombinant platelet adhesion inhibitor rLAPP. It is at present not quite possible to state with certainty which collagen, type I or III, is primarily involved in the increased reactivity. GPIa-IIa is a receptor for all collagens involved in adhesion in flow, whereas rLAPP inhibits adhesion to collagen types I, III, and IV. Recent data indicate that collagen types I and III are present in mixed collagen fibrils (59). The localization of collagen types I and III coincide, in accordance with their presence in the same fibrils. The area of increased reactivity for platelets corresponds with areas in which collagen types I and III are enriched, but there are also areas of the plaque where both collagens are enriched but no increased reactivity is seen. This suggests that changes in collagen types I and/or III are responsible for the increased reactivity, but these changes must involve more than increased local concentrations alone since the immunohistochemical studies indicate that areas with increased concentrations may occur without increased platelet reactivity. Further studies are required in which the collagens in the atherosclerotic plaque are more precisely localized and studied with regard to their ability to support platelet adhesion and aggregate formation. The composition of the collagen fibrils, and their association with other collagens, glycoproteins, or proteoglycans may be of crucial importance for the reactivity towards platelets.

\section{Acknowledgments}

We thank Dick van Wijchen (Department of Pathology, University Hospital Utrecht) for advice concerning the immunohistochemical studies, Drs. G. Jambroes, C. Klöpping, and N. de Jonge (Department of Cardiology, University Hospital Utrecht) for providing the fresh coronary arteries, Frits Kindt for his assistance in the preparation of the photographs, and Dr. M. Terlou (Department for Image Processing \& Design, Faculty of Biology, State University of Utrecht) for his assistance in the quantitative analysis. 
This study was supported by the Dutch Heart Foundation (88.066).

\section{References}

1. De Wood, M. A., J. Spores, R. Notske, L. T. Mouser, R. Burroughs, M. S. Golden, and H. T. Lang. 1980. Prevalence of total coronary occlusion during the early hours of transmural myocardial infarction. N. Engl. J. Med. 303:897-902.

2. Davies, M. J., and T. Thomas. 1981 . The pathological basis and microanatomy of occlusive thrombus formation in human coronary arteries. Philos. Trans. R. Soc. Lond. B Biol. Sci. 294:225-229.

3. Falk, E. 1983. Plaque rupture with severe pre-existing stenosis precipitating coronary thrombosis. Characteristics of coronary atherosclerotic plaques underlying fatal occlusive thrombi. Br. Heart J. 50:127-134.

4. Davies, M. J., and A. Thomas. 1984. Thrombosis and acute coronary artery lesions in sudden cardiac ischemic death. $N$. Engl. J. Med. 310:1137-1140.

5. Arbustini, E., M. Grasso, M. Diegoli, A. Pucci, M. Bramerio, D. Ardissino, L. Angoli, S. De Servi, E. Bramucci, A. Mussini, G. Minzioni, M. Vigano, and G. Specchia. 1991. Coronary atherosclerotic plaques with and without thrombus in ischemic heart syndromes: a morphologic, immunohistochemical, and biochemical study. Am. J. Cardiol. 68:36B-50B.

6. Kirk, J. E. 1962. Thromboplastin activities of human arterial and venous tissues. Proc. Soc. Exp. Biol. Med. 109:890-892.

7. Lyford, C. L., W. E. Connor, J. C. Hoak, and E. D. Warner. 1967. The coagulant and thrombogenic properties of human atheroma. Circulation. 36:284-293.

8. Byers, S. O., and M. Friedman. 1964. Contribution of atheromatous gruel to thrombus formation. Proc. Soc. Exp. Biol. Med. 115:436-438.

9. Jeynes, B. J., and B. A. Warren. 1981. Thrombogenicity of components of atheromatous material. An animal and in vitro model of cerebral atheroembolism. Arch. Pathol. Lab. Med. 105:353-357.

10. Wilcox, J. N., K. M. Smith, S. M. Schwartz, and D. Gordon. 1989. Localization of tissue factor in the normal vessel wall and in the atherosclerotic plaque. Proc. Natl. Acad. Sci. USA. 86:2839-2843.

11. Tipping, P. G., J. Malliaros, and S. R. Holdsworth. 1989. Procoagulant activity expression by macrophages from atheromatous vascular plaques. Atherosclerosis. 79:237-243.

12. Lawrence, J. B., L. G. Prevosti, W. S. Kramer, D. Y. Lu, and M. B. Leon. 1989. Platelet adherence and thrombus formation with flowing human blood on atherosclerotic plaque: reduced thrombogenicity of Watanabe-heritable hyperlipidemic rabbit aortic subendothelium. Thromb. Res. 54:99-114.

13. Groves, H. M., R. L. Kinlough-Rathbone, M. Richardson, L. Jorgensen, S. Moore, and J. F. Mustard. 1982. Thrombin generation and fibrin formation following injury to rabbit neointima. Studies of vessel wall reactivity and platelet survival. Lab. Invest. 46:605-612.

14. Sakariassen, K. S., P. A. M. M. Aarts, Ph.G. de Groot, W. P. M. Houdijk, and J. J. Sixma. 1983. A perfusion chamber developed to investigate platelet interaction in flowing blood with human vessel wall cells, their extracellular matrix, and purified components. J. Lab. Clin. Med. 102:522-535.

15. Nievelstein, P. F. E. M., P. A. D'Alessio, and J. J. Sixma. 1988. Fibronectin in platelet adhesion to human collagen types I and III. Studies in flowing blood using non fibrillar and fibrillar collagen. Arteriosclerosis. 8:200-206.

16. Turitto, V. T., and H. R. Baumgartner. 1987. Platelet-surface interaction. In Hemostasis and Thrombosis. Basic Principles and Clinical Practice. R. W. Colman, J. Hirsh, V. J. Marder, and E. W. Salzman, editors. J. B. Lippincott Company, Philadelphia. 562.

17. Verhoeven, A. J. M., M. E. Mommersteeg, and J. W. N. Akkerman. 1984. Metabolic energy is required in human platelets at any stage during optical aggregation and secretion. Biochim. Biophys. Acta. 800:242-250.

18. Adams, J. C. 1981. Heavy metal intensification of DAB-based HRP reaction product. J. Histochem. Cytochem. 29:775.

19. Stel, H. V., K. S. Sakariassen, B. J. Scholte, E. C. I. Veerman, Th. H. Van der Kwast, Ph.G. De Groot, J. J. Sixma, and J. A. Van Mourik. 1984. Characterization of 25 monoclonal antibodies to factor VII-von Willebrand factor: relationship between ristocetin-induced platelet aggregation and platelet adherence to subendothelium. Blood. 63:1408-1415.

20. Houdijk, W. P. M., Ph.G. De Groot, P. F. E. M. Nievelstein, K. S. Sakariassen, and J. J. Sixma. 1986. Subendothelial proteins and platelet adhesion: von Willebrand Factor and fibronectin, not thrombospondin, are involved in platelet adhesion to extracellular matrix of human vascular endothelial cells. Arteriosclerosis. 6:24-33.

21. Houdijk, W. P. M., K. S. Sakariassen, P. F. E. M. Nievelstein, and J. J. Sixma. 1985. Role of factor VIII-von Willebrand Factor and fibronectin in the interaction of platelets in flowing blood with monomeric and fibrillar collagen types I and III. J. Clin. Invest. 75:531-540.

22. Berndt, M. C., X. Du, and W. J. Booth. 1988. Ristocetin-dependent re- constitution of binding of von Willebrand Factor to purified human platelet membrane glycoprotein Ib-IX complex. Biochemistry. 27:633-640.

23. Sonnenberg, A., P. W. Modderman, and F. Hogervorst. 1988. Laminin receptor on the platelets is the integrin VLA-6. Nature (Lond.). 336:487-489.

24. Hindriks, G. A., M. J. W. IJsseldijk, A. Sonnenberg, and J. J. Sixma. 1992. Platelet adhesion to laminin: role of $\mathrm{Ca} 2+$ and $\mathrm{Mg} 2+$ ions, shear rate, and platelet membrane glycoproteins. Blood. 79:928-935.

25. Connolly, T. M., J. W. Jacobs, and C. Condra. 1992. An inhibitor of collagen-stimulated platelet activation from the salivary glands of the Haementeria officinalis leech. I. Identification, isolation, and characterization. J. Biol. Chem. 267:6893-6898.

26. Keller, P. M., L. D. Schultz, C. Condra, J. Karczewski, and T. M. Connolly. 1992. An inhibitor of collagen-stimulated platelet activation from the salivary glands of the Haementeria officinalis leech. II. Cloning of the cDNA and expression. J. Biol. Chem. 267:6899-6904.

27. Sakariassen, K. E., P. A. Bolhuis, and J. J. Sixma. 1980. Platelet adherence to subendothelium of human arteries in pulsatile and steady flow. Thromb. Res. 19:547-559.

28. Nieuwenhuis, H. K., J. W. N. Akkerman, W. P. M. Houdijk, and J. J. Sixma. 1985. Human blood platelets showing no response to collagen fail to express surface glycoprotein Ia. Nature (Lond.). 318:470-472.

29. Sixma, J. J., P. F. E. M. Nievelstein, J. J. Zwaginga, and Ph.G. De Groot. 1987. Adhesion of blood platelets to the extracellular matrix of cultured human endothelial cells. Ann. NY Acad. Sci. 516:39-51.

30. Gown, A. M., T. Tsukada, and R. Ross. 1986. Human atherosclerosis II. Immunocytochemical analysis of the cellular composition of human atherosclerotic lesions. Am. J. Pathol. 125:191-207.

31. Kocher, O., and G. Gabbiani. 1986. Cytoskeletal features of normal and atheromatous human arterial smooth muscle cells. Hum. Pathol. 17:875-880.

32. Hantgan, R. R., G. Hindriks, R. G. Taylor, J. J. Sixma, and P. G. De Groot. 1990. Glycoprotein Ib, von Willebrand Factor, and glycoprotein Ilb:Illa are all involved in platelet adhesion to fibrin in flowing whole blood. Blood. 76:345-353.

33. Agbanyo, F. R., J. J. Sixma, P. G. De Groot, L. R. Languino, and E. F. Plow. 1993. Thrombospondin-platelet interactions: role of divalent cations, wall shear rate and platelet membrane glycoproteins. J. Clin. Invest. 92:288-296.

34. Lawler, J., and E. R. Simons. 1983. Cooperative binding of calcium to thrombospondin: the effect of calcium on the circular dichoism and limitic tryptic digestion of thrombospondin. J. Biol. Chem. 258:12098-12101.

35. Saelman, E. U. M., H. K. Nieuwenhuis, K. M. Hese, Ph.G. De Groot, H. Sage, H. R. Gralnick, and J. J. Sixma. 1993. Platelet adhesion to collagen types I through VIII under conditions of stasis and flow is mediated by Gp Ia/IIa $\left(\alpha_{2} \beta_{1}\right.$ integrin). Blood. In press.

36. Santoro, S. A. 1986 . Identification of a 160,000 dalton platelet membrane protein that mediates the initial divalent cation-dependent adhesion of platelets to collagen. Cell. 46:913-920.

37. Weiss, H. J., V. T. Turitto, and H. R. Baumgartner. 1978. Effect of shear rate on platelet interaction with subendothelium in citrated and native blood. I. Shear rate dependent decrease of adhesion in von Willebrand's disease and the Bernard-Soulier syndrome. J. Lab. Clin. Med. 92:750-764.

38. Zwaginga, J. J., J. J. Sixma, and Ph.G. de Groot. 1990. Activation of endothelial cells induces platelet thrombus formation on their matrix. Studies of new in vitro thrombosis model with low molecular weight heparin as anticoagulant. Arteriosclerosis. 10:49-61.

39. Richardson, P. D., M. J. Davies, and G. V. R. Born. 1989. Influence of plaque configuration and stress distribution on fissuring of coronary atherosclerotic plaques. Lancet. 21:941-944.

40. Kragel, A. H., G. R. Shanthasundari, J. T. Wittes, and W. T. Roberts. 1989. Morphometric analysis of the composition of atherosclerotic plaques in the four major epicardial coronary arteries in acute myocardial infarction and in sudden coronary death. Circulation. 80:1747-1756.

41. Heras, M., J. H. Chesebro, W. J. Penny, K. R. Bailey, L. Badimon, and V. Fuster. 1989. Effect of thrombin inhibition on the development of acute plateletthrombus deposition during angioplasty in pigs. Circulation. 79:657-665.

42. Badimon, L., and J. J. Badimon. 1989. Mechanisms of arterial thrombosis in nonparallel streamlines. Platelet thrombi grow on the apex of stenotic severely injured vessel wall. J. Clin. Invest. 84:1134-1144.

43. Nichols, T. C., D. A. Bellinger, D. A. Tate, R. L. Reddick, M. S. Read, G. G. Koch, K. M. Brinkhous, and T. R. Griggs. 1990. Von Willebrand factor and occlusive arterial thrombosis. Arteriosclerosis. 10:449-461.

44. Wu, Q. Y., L. Drouet, J. L. Carrier, C. Rothschild, M. Berard, C. Rouault, J.P. Caen, and D. Meyer. 1987. Differential distribution of von Willebrand factor in endothelial cells. Comparison between normal pigs and pigs with von Willebrand disease. Arteriosclerosis. 7:47-54.

45. Kadsuda, S., Y. Okada, T. Minamoto, Y. Oda, Y. Matsui, and I. Nakanishi. 1992. Collagens in human atherosclerosis. Immunohistochemical analysis using collagen specific antibodies. Arterioscler. Thromb. 12:494-502.

46. McCullagh, K. G., V. C. Duance, and K. A. Bishop. 1980. The distribu- 
tion of collagen types I, III and V ( $\mathrm{AB}$ ) in normal and atherosclerotic human aorta. J. Pathol. 130:45-55.

47. Smith, E. B., and C. Ashall. 1986. Fibronectin distribution in human aortic intima and atherosclerotic lesions: concentration of soluble and collagenase-releasable fractions. Biochim. Biophys. Acta. 880:10-15.

48. Wight, T. N., G. J. Raugi, S. M. Mumby, and P. Bornstein. 1985. Light microscopic immunolocation of thrombospondin in human tissues. J. Histochem. Cytochem. 33:295-302.

49. Guettier, C., N. Hinglais, P. Bruneval, M. Kazatchkine, J. Bariety, and J.-P. Camilleri. 1989. Immunohistochemical localization of S protein/vitronectin in human atherosclerotic versus arteriosclerotic arteries. Virchows Arch. Pathol. Anat. Histol. 414:309-313.

50. Hayman, E. C., M. D. Pierschbacher, Y. Oehgren, and E. Ruoslahti. 1983. Serum spreading factor (vitronectin) is present at the cell surface and in tissues. Proc. Natl. Acad. Sci. USA. 80:4003-4007.

51. Bini, A., J. J. Fenoglio, R. Mesa-Tejada, B. Kudryk, and K. L. Kaplan. 1989. Identification and distribution of fibrinogen, fibrin, and fibrin ( ogen ) degradation products in atherosclerosis. Use of monoclaonal antibodies. Arteriosclerosis. 9:109-121.

52. Hantgan, R. R., S. C. Endenburg, I. Cavero, G. Marguerie, A. Uzan, J. J. Sixma, and Ph.G. De Groot. 1992. Inhibition of platelet adhesion to fibrin( ogen) in flowing whole blood by Arg-Gly-Asp and fibrinogen $\tau$-chain carboxy terminal peptides. Thromb. Haemostasis. 68:694-700.

53. Mumby, S. M., G. J. Raugi, and P. Bornstein. 1984. Interactions of thrombospondin with extracellular matrix proteins: selective binding to type $\mathrm{V}$ collagen. J. Cell Biol. 98:646-652.

54. Sussman, I. I., and J. H. Rand. 1982. Subendothelial deposition of van Willebrand factor requires the presence of endothelial cells. J. Lab. Clin. Med. 100:526-532.

55. Parsons, T. J., D. L. Haycraft, J. C. Hoak, and H. Sage. 1986. Interaction of platelets and purified collagens in a laminar flow model. Thromb. Res. 43:435443.

56. Bastida, E. B., G. Escolar, O. Ordinas, and J. J. Sixma. 1987. Fibronectin is required for platelet adhesion and for thrombus formation on subendothelium and collagen surfaces. Blood. 70:1437-1442.

57. Houdijk, W. P. M., and J. J. Sixma. 1985. Fibronectin in artery subendothelium is important for platelet adhesion. Blood. 65:598-604.

58. Baumgartner, H. R., and K. S. Sakariassen. 1985. Factors controlling thrombus formation on arterial lesions. Ann. NY Acad. Sci. 454:162-177.

59. Van der Rest, M., and R. Garrone. 1990. Collagens as multidomain proteins. Biochimie (Paris). 72:473-484. 